\title{
Deep Multistage Multi-Task Learning for Quality Prediction of Multistage Manufacturing Systems
}

\author{
Hao Yan ${ }^{1}$, Nurretin Dorukhan Sergin ${ }^{1}$, and William A. Brenneman ${ }^{2}$, \\ Stephen Joseph Lange ${ }^{3}$, and Shan $\mathrm{Ba}^{4}$ \\ ${ }^{1}$ School of Computing, Informatics, and Decision Systems Engineering, \\ Arizona State University, Tempe, Arizona \\ ${ }^{2}$ The Procter \& Gamble Company, Cincinnati, Ohio \\ ${ }^{3}$ ProcessDev, LLC, Cincinnati, OH \\ ${ }^{4}$ LinkedIn Corporation
}

\begin{abstract}
In multistage manufacturing systems, modeling multiple quality indices based on the process sensing variables is important. However, the classic modeling technique predicts each quality variable one at a time, which fails to consider the correlation within or between stages. We propose a deep multistage multi-task learning framework to jointly predict all output sensing variables in a unified end-to-end learning framework according to the sequential system architecture in the MMS. Our numerical studies and real case study have shown that the new model has a superior performance compared to many benchmark methods as well as great interpretability through developed variable selection techniques.
\end{abstract}

Keywords: deep neural network, multi-task learning, multistage manufacturing process, quality prediction

\section{Introduction}

A Multistage Manufacturing System (MMS) refers to a system consisting of multiple stages (e.g., units, stations, or operations) to fabricate a final product. In most cases, an MMS has a sequential configuration that links all stages with a directed flow from the initial part/material to the final product. In an MMS, massive data are collected from the in-situ multivariate sensing of process variables and the product quality measurements from intermediate stages and the final stage. It is challenging to model the MMS data due 
to the following characteristics: 1) Multiple correlated output variables: The MMS can have hundreds of output sensing variables at different stages that measure different quality attributes of the product in different stages. These quality responses are often correlated and evolve together with the product in the MMS. 2) High dimensional input variables: In an MMS, there can be hundreds of input variables (e.g., process sensors) in each stage as well as a large number of stages/stations. 3) Complex sequential dependency between stages and sensor measurements: The product quality from an MMS is determined by complex interactions among multiple stages. For example, the quality characteristics of one stage are not only influenced by local process variations within that stage, but also by the variation propagated from upstream stages.

The methodology developed in this paper is motivated by a diaper manufacturing system, which is composed of several sequential converting process steps with associated equipment components, which are controlled through many process factors. The setpoints for these factors and their variation may affect the reliability of the process and the quality of the product produced by it. Performance data of the converting equipment, as well as in-process product quality measures, are collected and stored by sensors and highspeed cameras installed on the production line. The time-series data may be high-frequency data (sampled every several milliseconds over seconds or minutes) and/or low-frequency data (samples collected on a oneminute frequency over months). The number of variables in this MMS is in the range of 500-1000. This data is used to detect abnormal performance early enough to prevent machine stops, quality degradation or scrap from rejected defective products. To model the variation propagation of the entire MMS, the stream of variation $(\mathrm{SoV})$ theory was proposed to model the variation propagation in an MMS (Shi, 2006). For example, when one of the stages of the multistage system experiences a malfunction, SoV can be used to model the consequence of such a change on the final product ( $\mathrm{Li}$ and Tsung, 2009; Lawless et al., 1999; Jin and Tsung, 2009). However, in modern manufacturing processes, such as semiconductor manufacturing or diaper manufacturing process, it is often not easy to define the system state explicitly due to the complex system mechanism. In conclusion, since classic approaches assume the design information and physical law of the process to be perfectly known, these classical approaches cannot be used for complex systems with unknown engineering or science knowledge.

In industrial practice and literature, to deal with more complicated types of data and nonlinear dependencies, predictive modeling techniques are often used. However, these models are typically designed based on a single output variable such as decision tree (Bakır et al., 2006, Jemwa and Aldrich, 2005) or neural network (Zhou et al., 2006; Tam et al., 2004, Chang and Jiang, 2002) and have the following major limitations: 1) Due to the independent modeling of each output variable at each stage, the correlation between each output variable is not considered. 2) Due to the need to have one model for every single variable, it lacks a unified way of modeling the MMS and variation propagation throughout the system. Hundreds or even thousands of 
independent models are needed, which is very hard to train and deploy. In addition, this greatly increases the number of parameters in modeling the entire MMS, which could lead to severe over-fitting when the number of samples is limited. 3) Due to the need for a unified model for process monitoring and control, there may be trade-offs between different output variables (e.g., quality variables). A joint modeling framework can be used for process optimization of multiple input variables, considering the trade-offs between different output variables simultaneously.

To address the aforementioned challenges, we aim to develop a data-driven approach, namely the deep multistage multi-task learning (DMMTL) framework, to link all the input and output variables in a sequential MMS. To the best of the authors' knowledge, this is the first end-to-end system-level predictive modeling framework in modeling all input and output variables jointly in an MMS. The proposed framework uses a latent state representation similar to the SoV model, but instead of specifying the hidden state representation manually, the proposed framework aims to learn the latent state representation and how it propagates through the MMS in an end-to-end fashion through all the output variables simultaneously. We further make many improvements on the model architecture to model the MMS, such as an independent state transition model and the group lasso penalty. Finally, since model interpretability and diagnostics are also important, we will also demonstrate how the proposed DMMTL is able to rank the most important input variables according to each output variable for system diagnostics.

To model multiple output variables simultaneously, the proposed DMMTL is also inspired by the recent development in multi-task learning (MTL). MTL is a sub-field of statistical machine learning in which multiple correlated learning tasks are solved at the same time while exploiting the commonalities across tasks while modeling their differences (Caruana, 1997). The proposed DMMTL can benefit through incorporating the use of MTL to jointly model all output variables from all stages simultaneously since these output variables often measure correlated attributes of the same product in the MMS. However, MTL by itself can only be used for joint modeling of multiple sensing variables within the same stage, which fails to model the out variables with sequential order.

Finally, we present an overview of the proposed methodology compared to existing manufacturing methodology such as stream of variation (denoted as "SoV") (Shi, 2006), predictive modeling (denoted as "Predictive") (Kuhn and Johnson, 2013), and multi-task learning (denoted as "MTL") (Obozinski et al., 2006) in Table 1. The proposed method is the only one that can handle the unknown physical state (e.g., unknown transition functions), nonlinear models, variable selection, joint multistage modeling, and joint multiple sensor modeling within the stages. Finally, the proposed method can also achieve diagnostics and interpretability by using the gradient tracking techniques developed in this paper.

The remaining parts of the paper are organized as follows. Section 2 reviews the related literature in 
Table 1: Comparison of Literature

\begin{tabular}{|l|l|l|l|l|}
\hline & Proposed & SoV & Predictive & MTL \\
\hline Unknown Physical State Definition & $\checkmark$ & $\times$ & $\checkmark$ & $\checkmark$ \\
\hline Nonlinear Extension & $\checkmark$ & $\times$ & $\checkmark$ & $\times$ \\
\hline Variable Selection & $\checkmark$ & $\times$ & $\checkmark$ & $\checkmark$ \\
\hline Sequential Multistage Modeling & $\checkmark$ & $\checkmark$ & $\times$ & $\times$ \\
\hline $\begin{array}{l}\text { Joint Multi-sensor Modeling } \\
\text { Within Stages }\end{array}$ & $\checkmark$ & $\checkmark$ & $\times$ & $\times$ \\
\hline Diagnostics & $\checkmark$ & $\checkmark$ & $\times$ & $\times$ \\
\hline
\end{tabular}

manufacturing modeling. In Section 3, we develop the proposed methodology for quality prediction and variable selection. Section 4 discusses the estimation procedure and variable selection techniques. Section 5 presents a simulation study to compare the proposed framework with several traditional methods in the literature. In Section 6, we apply the proposed framework to model a diaper manufacturing line. In Section 7 . we provide concluding remarks and future direction. For a more detailed comparison of the proposed method with SoV, please refer to Appendix A.

\section{Literature Review}

In this section, we will review the related literature in the modeling of manufacturing systems. We briefly classify existing techniques into two types, single-stage modeling and multistage modeling.

Single-stage models typically focus on process monitoring (Joe Qin, 2003, Kourti et al., 1996, MacGregor and Kourti, 1995) and the prediction (Hao et al. 2016, Jin et al., 2007) of sensing variables observed in the same stage of the manufacturing system. For process monitoring, uni-variate (Shewhart, 1931), multivariate (Lowry and Montgomery, 1995), profile-based (Woodall, 2007), multi-channel-profile-based (Paynabar et al., 2013 Zhang et al., 2018), and image-based (Yan et al., 2015, 2017) process monitoring techniques are developed. For quality prediction, regression and classification techniques such as linear regression (Skinner et al., 2002), logistic regression (Jin et al., 2007), tensor learning (Yan et al., 2018), decision trees (Bakır et al., 2006, Jemwa and Aldrich, 2005), and neural network (Zhou et al., 2006, Tam et al., 2004, Chang and Jiang, 2002) are applied to relate the input variables (e.g. process sensing variables) with the output variables (e.g. quality sensing variables). Despite the use of nonlinear methods and the ability to incorporate heterogeneous high-dimensional data, it still lacks a unified framework for modeling the variation propagation among stages.

To model multistage systems, SoV has been successfully implemented in the multistage automotive assembly process (Jin and Shi, 1999, Apley and Shi, 1998, Ceglarek and Shi, 1996, Ding et al., 2005, Shiu et al. 1997) and machining process (Huang et al., 2002, Liu et al., 2009, Zhou et al., 2003; Abellan-Nebot 
et al., 2012). For example, SoV introduces the state space representation to quantify the system status. In a traditional SoV model, the state variables are defined physically (e.g., geometry deviation of the product (Ding et al. 2005)). For a more detailed literature review on MMS models, please refer to (Shi, 2006). There are some other techniques besides SoV that have been developed for multistage modeling. Bayesian network techniques (Friedman et al. 1997, Jensen, 1996) have been proposed to model the complex dependency between multiple manufacturing stages for both process monitoring (Liu et al., 2014, Yu and Rashid, 2013, Liu and Shi, 2013) and quality prediction (Reckhow, 1999, Correa et al., 2008). However, these techniques assume that the complex dependencies between multiple output variables are known and require the feature selection techniques to be used beforehand, and thus cannot be applied to a system with unknown transition and dependency. In the literature, reconfigured piece-wise linear regression trees are developed (Jin and Shi, 2012 to take advantage of intermediate quality variables and model the nonlinear relationship between the sequential order of the input and output variables. However, this technique cannot perform feature selection for a large number of sensors and it also assumes the same quality responses are measured in the intermediate stages. Furthermore, it optimizes the model in a greedy stage-wise approach, which may suffer from local optimality. In conclusion, similar to SoV, most of the techniques in the literature focus on either manual selection of important sensors, manual extraction of useful features transformation, and clear definition of system state and transition before the MMS modeling can be applied. But these techniques cannot be used for quality prediction of complex systems with unknown architectures (Ding et al., 2005, Apley and Shi, 1998; Zhou et al., 2003, 2004; Ceglarek and Shi, 1996).

\section{Methodology Development}

In this section, we will first define the problem setting and notations in Section 3.1 followed by our proposed DMMTL framework in Section 3.2 We further derived a more efficient optimization algorithm to handle the non-smooth loss function and penalty in Section 3.3 .

\subsection{Problem Setting and Notation}

We denote $\mathbf{x}_{k}=\left(\mathbf{x}_{k, 1}, \cdots, \mathbf{x}_{k, n_{x, k}}\right)^{T}$ is a vector of input variables (i.e. process sensing variables) in stage $k$, where $x_{k, i}$ is the $i^{t h}$ sensing variable in stage $k$, and $n_{x, k}$ is the total number of input sensing variables in stage $k=1, \cdots, K$, where $K$ is the number of stages. We denote $\mathbf{y}_{k}=\left(y_{k, 1}, \cdots, y_{k, n_{y, k}}\right)^{T}$ as the output variables (i.e. product quality sensing variables) in stage $k$, where $y_{k, j}$ is the $j^{\text {th }}$ sensing variable in stage $k$ and $n_{y, k}$ is the total number of output sensing variables in stage $k$. To link multiple stages together, we introduce the hidden state variable $\mathbf{h}_{k}$, which is a vector to represent the state of the product in stage 
$k$. For simplicity, we assume the hidden state variable $\mathbf{h}_{k}$ is of the same dimension $n_{h}$ across different states. The goal of this research is to build a multi-task learning framework to predict the quality indices $\mathcal{Y}=\left\{\mathbf{y}_{1}, \cdots, \mathbf{y}_{K}\right\}$ measured at different stages given $\mathcal{X}=\left\{\mathbf{x}_{1}, \cdots, \mathbf{x}_{K}\right\}$. In this section, we assume that we are only dealing with data with sample size 1 . We will discuss how to extend this to the mini-batch version utilizing mini-batch stochastic gradient descent with multiple samples in Appendix D in detail.

\subsection{Proposed Framework}

We will introduce our proposed DMMTL model to solve the aforementioned challenges by learning the hidden state representation $\mathbf{h}_{k}$ from data. Here, $\mathbf{h}_{k}$ should contain the information not only to predict the current state output $\mathbf{y}_{k}$ but also the future stage output $\mathbf{y}_{k^{\prime}}$ with $k^{\prime}>k$. In $k^{t h}$ manufacturing stage, we will define the transition function to model the state transition between $\mathbf{h}_{k-1}$ and $\mathbf{h}_{k}$ and the emission function to model the relationship between $\mathbf{h}_{k}$ and $\mathbf{y}_{k}$ as learnable parametric functions with model parameters $\boldsymbol{\theta}_{k}^{h}, \boldsymbol{\theta}_{k}^{g}$ as

$$
\mathbf{h}_{k}=f_{k}\left(\mathbf{h}_{k-1}, \mathbf{x}_{k} ; \boldsymbol{\theta}_{k}^{h}\right), \mathbf{y}_{k}=g_{k}\left(\mathbf{h}_{k} ; \boldsymbol{\theta}_{k}^{g}\right)+\epsilon_{y k},
$$

$\epsilon_{y k} \sim N\left(0, \sigma^{2}\right)$. One example of such architecture is listed in 12 , which we use a one-layer neural network to model the nonlinear state transition and emission function.

$$
\mathbf{h}_{k}=\sigma\left(\mathbf{W}_{x k} \mathbf{x}_{k}+\mathbf{U}_{h k} \mathbf{h}_{k-1}+\mathbf{b}_{h k}\right), \mathbf{y}_{k}=\mathbf{V}_{y k} \mathbf{h}_{k}+\mathbf{b}_{g k}+\epsilon_{y k},
$$

where $\sigma(x)=1 /(1+\exp (-x))$ is the activation function. Define the stage transition model parameters as $\boldsymbol{\theta}_{k}^{h}=\left\{\mathbf{U}_{h k}, \mathbf{W}_{x k}, \mathbf{b}_{h k}\right\} \cdot g_{k j}(\cdot)$ represents the emission function to link the hidden variable to the output variable. For example, $g_{k j}(\cdot)$ can be a linear function with model parameters $\boldsymbol{\theta}_{k}^{g}=\left\{\mathbf{V}_{y k}, \mathbf{b}_{g k}\right\}$ or nonlinear functions such as neural networks. Here, we denote $\boldsymbol{\theta}_{k}=\left\{\boldsymbol{\theta}_{k}^{h}, \boldsymbol{\theta}_{k}^{g}\right\}$ as the model parameters for stage $k$. Furthermore, if we use one-layer neural networks for both transition and emission, the model does share some similarity with RNN models. However, the major limitation of using RNN in MMS is that different manufacturing stages are inherently different. The underlying physics is entirely different for each stage which not only results in the different transition parameters $\boldsymbol{\theta}_{k}^{h}=\left\{\mathbf{U}_{h k}, \mathbf{W}_{x k}, \mathbf{b}_{h k}\right\}$ and emission parameters $\boldsymbol{\theta}_{k}^{g}=\left\{\mathbf{V}_{y k}, \mathbf{b}_{g k}\right\}$. RNN also assumes the same set of variables are predicted in each time. However, in MMS, different quality inspection sensors are set up in each manufacturing stage, denoted by $\mathbf{y}_{k}$. Finally, RNN is a complicated model and can not achieve input and output variable selection as the proposed approach. More discussion of the relationship and differences of the one-layer version of the proposed DMMTL and RNN are shown in Appendix B. 
The benefit of the proposed method is also its ultimate flexibility of plugging in any differentiable functions as $f_{k}(\cdot)$ and $g_{k}(\cdot)$. For example, depending on different applications, we can either use simpler models (e.g., linear models) or more complicated models (e.g., deep neural networks). As an example, two-layer transition and emission networks are shown as follows:

$$
\begin{aligned}
& \mathbf{h}_{k}=\sigma\left(\mathbf{U}_{h k}^{2} \mathbf{h}_{k}^{1}+\mathbf{b}_{h k}^{2}\right), \mathbf{h}_{k}^{1}=\sigma\left(\mathbf{W}_{x k} \mathbf{x}_{k}+\mathbf{U}_{h k}^{1} \mathbf{h}_{k-1}^{2}+\mathbf{b}_{h k}^{1}\right) \\
& \mathbf{y}_{k}=\mathbf{V}_{y k}^{2} \mathbf{y}_{k}^{1}+\mathbf{b}_{g k}^{2}+\epsilon_{y k}, \mathbf{y}_{k}^{1}=\sigma\left(\mathbf{V}_{y k}^{1} \mathbf{h}_{k}+\mathbf{b}_{g k}^{1}\right)
\end{aligned}
$$

In this case, the stage transition model parameters are $\boldsymbol{\theta}_{k}^{h}=\left\{\mathbf{U}_{h k}^{1}, \mathbf{U}_{h k}^{2}, \mathbf{W}_{x k}, \mathbf{b}_{h k}^{1}, \mathbf{b}_{h k}^{2}\right\}$, and the emission parameters $\boldsymbol{\theta}_{k}^{g}=\left\{\mathbf{V}_{y k}^{1}, \mathbf{V}_{y k}^{2}, \mathbf{b}_{g k}^{1}, \mathbf{b}_{g k}^{2}\right\}$ and $\boldsymbol{\theta}_{k}=\left\{\boldsymbol{\theta}_{k}^{h}, \boldsymbol{\theta}_{k}^{g}\right\}$. In general, we can use a neural network with depth $D_{1}$ to model for the transition function and a neural network with depth $D_{2}$ to for the emission function. In this case, the model parameter is $\boldsymbol{\theta}_{k}=\left\{\mathbf{W}_{x k},\left\{\mathbf{U}_{h k}^{d}, \mathbf{b}_{h k}^{d}\right\}_{d=1, \cdots, D_{1}},\left\{\mathbf{V}_{y k j}^{d}, \mathbf{b}_{g k}^{d}\right\}_{d=1, \cdots D_{2}}\right\}$. To show the relationship of the proposed framework and the deep neural network, we also visualized the architecture of the proposed methods in Figure 1. In this figure, we showed a special case where only a single layer neural network is used for the transition between the hidden variables. In this case, the number of transition layers is exactly the number of manufacturing stages. There are some additional layers models the relationship of the input variables/output variables with the hidden variables.

In the proposed DMMTL, instead of modeling each $P\left(y_{k j} \mid\left\{\mathbf{x}_{1}, \cdots, \mathbf{x}_{k}\right\}\right)$ individually, we assume that the hidden state $\mathbf{h}_{k}$ is learned through the model such that it compresses all the necessary information to predict the current stage output $\mathbf{y}_{k}$ and future stage output $\mathbf{y}_{k^{\prime}}$ for $k^{\prime}>k$. There are two benefits of using the latent variable $\mathbf{h}_{k}$ rather than the original data $\left.\left\{\mathbf{x}_{1}, \cdots, \mathbf{x}_{k}\right\}: 1\right)$ Dimension reduction in the sequential transition model: $\left\{\mathbf{x}_{1}, \cdots, \mathbf{x}_{k}\right\}$ is typically very high-dimension, especially for the later stages when $k$ is large. Here the model creates the low-dimensional hidden state variable $\mathbf{h}_{k}$ to compress all the necessary information from $\left\{\mathbf{x}_{1}, \cdots, \mathbf{x}_{k}\right\}$. Therefore, the conditional probability can be compactly represented by $P\left(y_{k j} \mid\left\{\mathbf{x}_{1}, \cdots, \mathbf{x}_{k}\right\}\right)=P\left(y_{k j} \mid \mathbf{h}_{k}\right)$. 2) Shared representation for multi-task learning: Here $\mathbf{h}_{k}$ itself is used to predict all the output variables $y_{k j}$ in stage $k$, which is especially helpful when the output variables $y_{k j}$ in each stage $k$ are correlated. By assuming that different output variables are conditionally independent given the hidden state variables $\mathbf{h}_{k}$, the architecture of the model is shown in Figure 2

The benefit of introducing this recursive structure and the hidden state representation $\mathbf{h}_{k}$ is that the negative joint log likelihood $\mathcal{L}(\boldsymbol{\Theta} ; \mathcal{X}, \mathcal{Y})$ can be decomposed in each stage $k$ and each sensor $j$ as

$$
\mathcal{L}(\boldsymbol{\Theta} ; \mathcal{X}, \mathcal{Y})=-\sum_{k=1}^{K} \log P\left(\mathbf{y}_{k} \mid \mathbf{h}_{k} ; \boldsymbol{\Theta}\right)=-\sum_{k=1}^{K} \sum_{j=1}^{n_{y, k}} \log P\left(y_{k j} \mid \mathbf{h}_{k} ; \boldsymbol{\Theta}\right) \propto \sum_{k=1}^{K} \sum_{j=1}^{n_{y, k}} L_{k}\left(\mathbf{e}_{k} ; \Theta\right)
$$




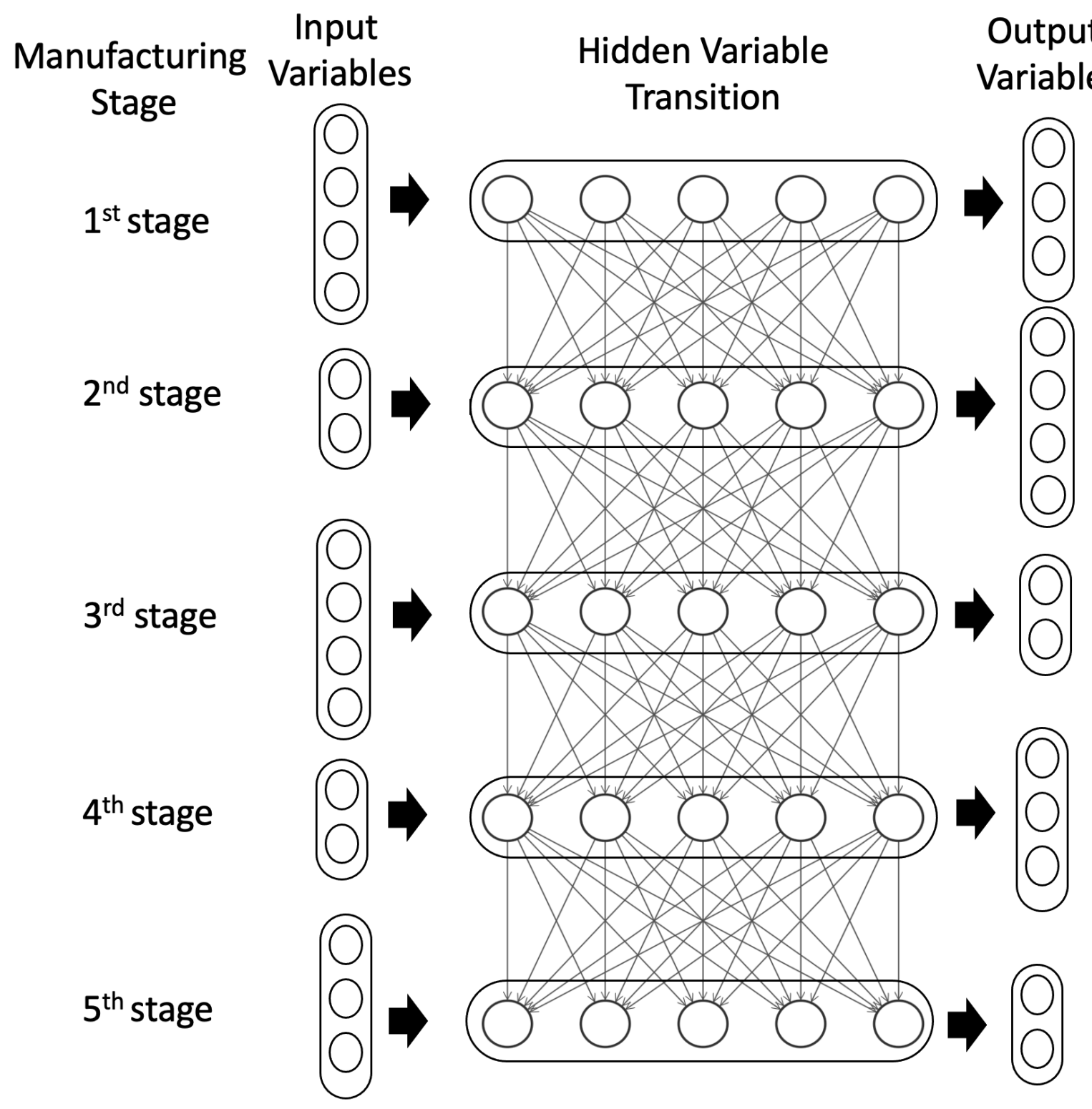

Figure 1: Deep Neural Network Structure for the Proposed Method 


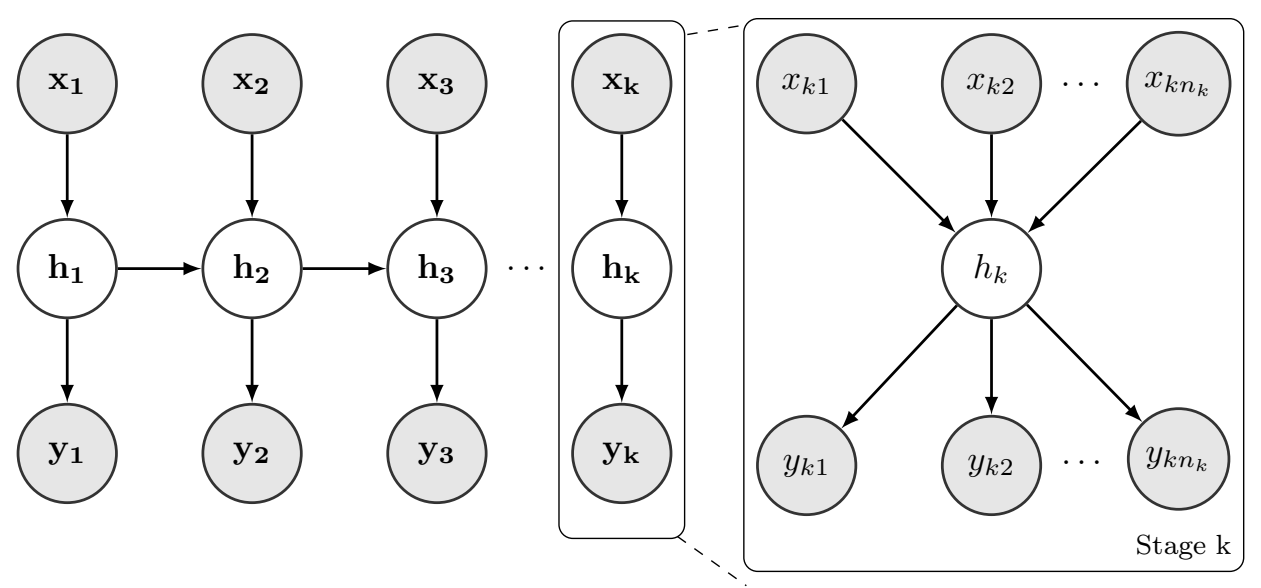

Figure 2: Architecture of the Proposed DMMTL

where $e_{k j}=y_{k j}-g_{k j}\left(\mathbf{h}_{k} ; \boldsymbol{\Theta}\right)$ and $L_{k}(\cdot)$ is the negative log-likelihood of the noise distribution. For example, for Gaussian noise, we can set $L_{k}\left(\mathbf{e}_{k} ; \Theta\right)=\left\|\mathbf{e}_{k}\right\|^{2}$. We will discuss in more detail how to define $L_{k}\left(\mathbf{e}_{k} ; \Theta\right)$ in MMS. Finally, to make the model interpretable and to prevent overfitting, we propose to minimize the following loss function:

$$
\min _{\Theta} \mathcal{L}(\Theta)+\mathcal{R}(\Theta)
$$

where $\Theta=\left\{\boldsymbol{\theta}_{1}, \cdots, \boldsymbol{\theta}_{K}\right\} . \mathcal{L}(\Theta)=\sum_{k=1}^{K} L_{k}\left(\boldsymbol{\theta}_{k}\right)$ is the likelihood loss function, $\mathcal{R}(\Theta)=\sum_{k=1}^{K} R_{k}\left(\boldsymbol{\theta}_{k}\right)$ is the regularization function. Here, $R_{k}\left(\boldsymbol{\theta}_{k}\right)$ and $L_{k}\left(\boldsymbol{\theta}_{k}\right)$ are defined as the regularization term and the loss function in stage $k$, which will be defined in more detail later. Equation (5) can also be seen as a multi-layer neural network, where the architecture of the neural network structure depends on the physical layout of the manufacturing system. For example, each layer (or a set of layers) represents one stage of the manufacturing system, with the emission network output the prediction of output sensing variables (i.e., quality indices measured at each stage), and the transmission network passes the information to the next stage. We propose to combine the process variables $\mathbf{x}_{k}$ or quality variables $\mathbf{y}_{k}$ for all $K$ stages in a multi-task learning framework in order to optimize $\boldsymbol{\theta}_{k}$ in an end-to-end fashion.

It is also interesting to compare the proposed DMMTL in Equation (5) to the independent modeling approach, where each output variable $y_{k j}$ at stage $k$ and output $j$ is modeled independently. Because such an independent modeling approach needs to introduce new model parameters $\boldsymbol{\theta}_{k j}$ for each output variable $j$ in each stage $k$, for a $K$-stage system with $n_{y}$ output sensors and $n_{x}$ input sensors in each stage, it requires to have $O\left(K^{2} n_{x} n_{y}\right)$ model parameters in total. These model parameters are normally time-consuming to train and can lead to the over-fitting problem. On the other hand, since our proposed framework reduces the hidden state dimension to $n_{h}$ using $\boldsymbol{\theta}_{k}^{h}, k=1, \cdots, K$ with $O\left(K n_{h}\left(n_{x}+n_{y}\right)\right)$ number of variables, which is typically much smaller than $O\left(K^{2} n_{x} n_{y}\right)$ given $n_{h} \ll \min \left(K n_{x}, K n_{y}\right)$. Therefore, a shared representation 
yields a more compact representation with better memory efficiency.

Furthermore, we would like to propose the loss function $L_{k}(\cdot)$ and regularization $R_{k}\left(\boldsymbol{\theta}_{k}\right)$ in Equation (1) that leads to a better engineering interpretation. In addition, we use the regularized model to select key input variables and output variables. More specifically, we propose the group lasso penalty (Yuan and Lin, 2006) and robust statistics for a more interpretable transition model, allowing us to perform input and output variable selection.

Group Sparsity Penalty In MMS, many input variables are irrelevant in relation to predicting output variables. Therefore, these input variables should not affect the hidden states. To select the important sensors in each stage, we propose to use the $L_{2,1}$ nom on $\mathbf{W}_{x k}^{T}$ to encourage the model to only select the most important sensors. In the literature, the $L_{2,1}$ norm is defined as $\|\mathbf{W}\|_{2,1}=\sum_{i} \sqrt{\sum_{j} W_{i j}^{2}}$, which penalizes the entire row of the matrix $\mathbf{W}$ to be zero. Here, we propose to add this $L_{2,1}$ norm on the transposed coefficient $\mathbf{W}_{x k}$ on (4), which penalizes the entire column of matrix $\mathbf{W}_{x k}$ to be zero. In other words, if we define the $i^{\text {th }}$ column of $\mathbf{W}_{x k}$ is $\mathbf{w}_{i, x k},\left\|\mathbf{W}_{x k}^{T}\right\|_{2,1}=\sum_{j}\left\|\mathbf{w}_{i, x k}\right\|$.

This penalty not only controls the model flexibility but also can lead to a more interpretable result due to the sensor selection power as follows:

$$
R_{k}\left(\boldsymbol{\theta}_{k}\right)=\lambda_{x}\left\|\mathbf{W}_{x k}^{T}\right\|_{2,1}+\frac{\lambda}{2}\|\Theta\|^{2}
$$

where $\|\cdot\|_{2}$ is the $L_{2}$ norm and $\left\|\mathbf{W}_{x k}^{T}\right\|_{2,1}$ tends to penalize the entire column of $\mathbf{W}_{x k}$ to be 0 . For example, if the $i^{\text {th }}$ column of $\mathbf{W}_{x k}$ is 0 , the $i^{t h}$ input variable of $x_{k i}$ in stage $k$ is not selected, which means this input variable $x_{k i}$ is not important to the prediction of any output variables in the following stages. Furthermore, $\frac{\lambda}{2}\|\Theta\|^{2}$ is added for the general $L_{2}$ penalty to prevent overfitting. We will discuss how to identify the most important input variable for each individual output variable in Section 4 . These regularization terms enforce that only some of the input variables or hidden variables are used in the prediction, which models the weakly correlated patterns and improves the interpretability of the model. Finally, we will use the validation prediction error to select the best tuning parameters $\lambda_{x}, \lambda$ and $\gamma$.

Robust Regression The most commonly defined loss function is the sum of square error (SSE) loss defined as $L_{k}(\mathbf{e})=\|\mathbf{e}\|^{2}$. However, the proposed DMMTL is a multi-task learning framework that focuses on predicting multiple tasks (i.e., quality variables) simultaneously. In reality, due to the lack of sensing powers, many output variables simply cannot be predicted by the input variables no matter what models we use. Using these non-informative quality variables may not help or even corrupt the training results. Here, the goal is to derive the loss function such that the model is robust to these unrelated tasks or achieve a 
better balance between tasks. Therefore, we will compare the use of the Huber loss function $L_{k}(e)=\rho(e)$ as defined in Equation (7) with the traditional sum of square loss function.

$$
\rho(e)=\left\{\begin{array}{ll}
\|e\|^{2} & |e| \leq \frac{\gamma}{2} \\
\gamma|e|-\frac{\gamma^{2}}{4} & |e|>\frac{\gamma}{2}
\end{array} .\right.
$$

Huber loss can be used instead of the mean-squared error. The Huber loss function uses a linear function when the difference is large which enables a more robust estimation. Furthermore, we find that it can also help the model identify and focus more directly on the related output variables by being more robust to the unrelated output variables. We will discuss how to optimize the model parameters efficiently in Section 3.3

\subsection{Optimization Algorithm}

It is worth noting that problem [5] has a non-smooth loss function penalty such as $\lambda_{x}\left\|\mathbf{W}_{x k}^{T}\right\|_{2,1}$. In literature, the stochastic sub-gradient algorithm can be used to handle the non-smooth penalty. However, the convergence speed of the stochastic sub-gradient algorithm is typically slow. Therefore, to address the nonsmooth loss function, we propose to combine the stochastic proximal gradient algorithm and block coordinate algorithm for efficient optimization.

To efficiently optimize the problem, we will discuss the use of the stochastic proximal gradient as follows. First, we will first establish the equivalency of the proposed algorithm by introducing another set of outlier variables $\mathcal{A}=\left\{a_{k, j}\right\}_{k, j}$. Here, $a_{k, j}$ represents the outlier in stage $k$ and sensor $j$. If $a_{k, j}=0$, it implies that there is no outlier in stage $k$ and sensor $j$ for the current sample. However, if $a_{k, j} \neq 0$, it implies that the outlier occurs for this sample.

Proposition 1. Solving $\boldsymbol{\Theta}$ in (5) will give the same solution as solving $\boldsymbol{\Theta}$ in (8).

$$
\min _{\Theta,\left\{a_{k j}\right\}} \mathcal{L}(\Theta, \mathcal{A})+\lambda_{x}\left\|\mathbf{W}_{x k}^{T}\right\|_{2,1}+\frac{\lambda}{2}\|\Theta\|^{2}+\gamma \sum_{k, j}\left\|a_{k, j}\right\|_{1}
$$

where $\mathcal{L}(\Theta, \mathcal{A})=\sum_{k, j}\left\|y_{k j}-g_{k j}\left(\mathbf{h}_{k} ; \boldsymbol{\Theta}\right)-a_{k j}\right\|^{2}$ is the loss function, $\mathcal{A}=\left\{a_{k, j}\right\}_{k, j}$.

It worth noting that both $y_{k j}$ and $a_{k j}$ are scalar if only a single sample is used. However, if multiple samples are used, $y_{k j}$ and $a_{k j}$ are vectors. Please see Appendix D for more details to generalize the proposed methods to the mini-batch version.

The proof relies on the equivalency of the Huber loss function and the sparse outlier decomposition and has been proved in (Mateos and Giannakis, 2011). The benefit of using $(8)$ is that $\mathcal{R}(\Theta, \mathcal{A})$ is continuous and 
differentiable so that the back-propagation algorithm can be used efficiently. We will show how to handle non-differentiable components of $\sum_{k, j}\left\|a_{k j}\right\|_{1}$ and $\left\|\mathbf{W}_{x k}^{T}\right\|_{2,1}$ by using Block Coordinate algorithm to update each set of variables $\mathcal{A}, \mathbf{W}_{x k}$, and $\Theta$ (excluding $\mathbf{W}_{x k}$ ) iteratively until convergence. Proposition 2 shows how to solve $\mathcal{A}$ and $\mathbf{W}_{x k}$, given the other variables are fixed.

Proposition 2. In the $t^{\text {th }}$ iteration, solving $a_{k j}$ given $\Theta=\Theta^{(t)}$ in (8) can be derived analytically as

$$
a_{k j}^{(t)}=S_{\gamma / 2}\left(y_{k j}-g_{k j}\left(\mathbf{h}_{k} ; \boldsymbol{\Theta}^{(t)}\right)\right) .
$$

Given $\Theta=\Theta^{(t)}$ and $\mathcal{A}^{(t)}$, the upper bound of (8), defined as

$$
\begin{aligned}
& \min _{\left\{\mathbf{w}_{i, x k}\right\}} \mathcal{L}\left(\Theta^{(t-1)}, \mathcal{A}^{(t)}\right)+\sum_{i, k} \frac{\partial \mathcal{L}\left(\Theta^{(t-1)}, \mathcal{A}^{(t)}\right)}{\partial \mathbf{w}_{i, x k}}\left(\mathbf{w}_{i, x k}-\mathbf{w}_{i, x k}^{(t-1)}\right) \\
& +\frac{L}{2} \sum_{i, k}\left\|\mathbf{w}_{i, x k}-\mathbf{w}_{i, x k}^{(t-1)}\right\|^{2}+\lambda_{x} \sum_{i, k}\left\|\mathbf{w}_{i, x k}\right\|_{2}+\frac{\lambda}{2} \sum_{i, k}\left\|\mathbf{w}_{i, x k}\right\|^{2} .
\end{aligned}
$$

in the proximal gradient algorithm, can be solved by

$$
\mathbf{w}_{i, x k}^{(t)}=S_{\frac{\lambda x}{L+\lambda}}\left(\frac{L}{L+\lambda}\left(\mathbf{w}_{i, x k}^{(t-1)}-\frac{1}{L} \frac{\partial \mathcal{L}\left(\Theta^{(t)}, \mathcal{A}^{(t)}\right)}{\partial \mathbf{w}_{i, x k}}\right)\right) .
$$

Here, $S_{\gamma}(x)=\operatorname{sgn}(x)(|x|-\gamma)_{+}$is the soft thresholding operator, in which $\operatorname{sgn}(x)$ is the sign function and $x_{+}=\max (x, 0) . \Theta^{(t)}, \mathcal{A}^{(t)}$, and $\mathbf{w}_{i, x k}^{(t)}$ are the corresponding values of the model parameters in the $t^{t h}$ iterations. $L$ is the Lipschitz constance of the function $\mathcal{L}(\cdot)$.

The proof is given in Appendix $\mathrm{C}$ The gradient $\frac{\partial \mathcal{L}\left(\Theta^{(t)}, \mathcal{A}^{(t)}\right)}{\partial \mathbf{w}_{i, x k}}$ can be computed from the back-propagation algorithm, which is detailed in Appendix D. Finally, since the loss function is differential for the parameter $\tilde{\Theta}$, defined as $\Theta$ excluding $\mathcal{W}=\left\{W_{i, x k}\right\}_{i, k}$, the standard stochastic gradient algorithm can be applied given $\mathcal{W}=\mathcal{W}^{(t)}$ and $\mathcal{A}=\mathcal{A}^{(t)}$ as follows:

$$
\tilde{\Theta}=\tilde{\Theta}-c \frac{\partial \mathcal{L}\left(\Theta, \mathcal{W}^{(t)}, \mathcal{A}^{(t)}\right)}{\partial \tilde{\Theta}}
$$

where $c$ is the step length. Finally, the mini-batch version of the algorithm can also be derived with a subset of samples $\left\{\mathcal{X}^{n}, \mathcal{Y}^{n}\right\}_{n \in \mathcal{N}^{t}}$ in iteration $t$. More details of using stochastic optimization algorithm is also shown in Appendix D.

The algorithm is summarized in Algorithm 1. Given the non-convex formulation of deep neural networks, there is no guarantee that the algorithm would converge to the the global optimum. However, we find out that optimization algorithms typically perform reasonably well. In case the training failed to converge to 
a good optimum (measured by the validation accuracy), the training can be restarted with a new random initialization point.

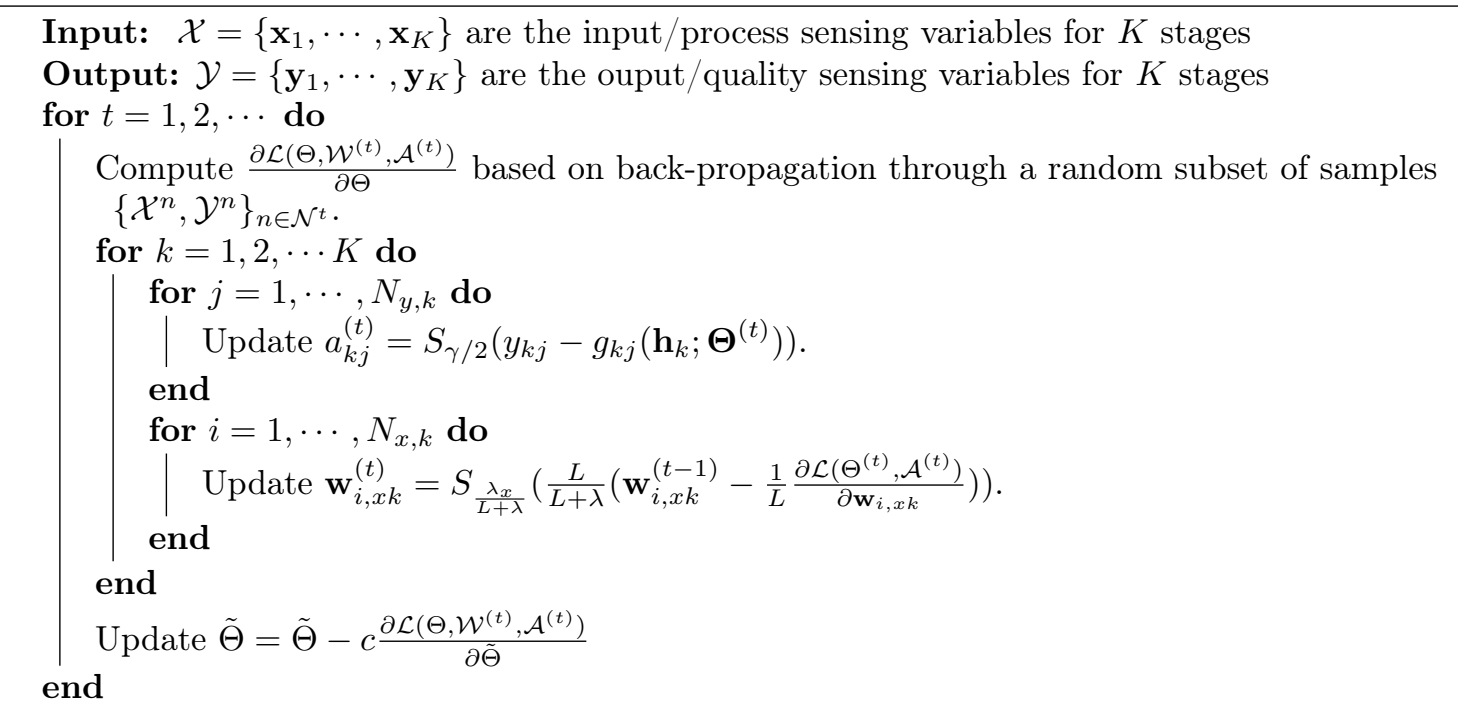

Algorithm 1: Optimization Algorithm for DMMTL

\subsection{Tuning Parameter Selection}

In this section, we would like to discuss the selection of tuning parameters. Overall, we need to decide the following tuning parameters: 1) Number of dimensions of the hidden vector $\mathbf{h}_{k}$. For the dimensionality of the hidden vector $\mathbf{h}_{k}$, in principle, we can vary the number of neurons for $\mathbf{h}_{k}$ for different stage $k$. Here, we would like to mention that in this paper, we use the same dimensionality of the hidden vector $\mathbf{h}_{k}$ for different stage $k$ for simplicity. However, we will use the regularization term to control the amount of information that flows into the network. 2) Tuning parameter $\lambda_{x}$ and $\lambda$. Here, the $\lambda_{x}$ is used to control the sparsity of the input variables. For example, increasing $\lambda_{x}$ will lead to a more sparse selection of the input variables at different stages. $\lambda$ is used to regularize the $L_{2}$ norm of all the parameters. 3) The depth of neural network architectures for each stage. Again, in principle, we can use different layer depths for different manufacturing stages. For example, if one particular manufacturing stage is more complex, we can actually increase the number of layers for such a stage. In our simulation study and case study, we find that a one-layer neural network for each stage is enough to cover most of the cases.

Finally, to select all these parameters, we propose to use a set of validation dataset $\left\{\mathcal{X}^{\text {val }}, \mathcal{Y}^{\text {val }}\right\}$. Furthermore, we will use a randomized search of the tuning parameter space with the prediction accuracy of the validation set $\left\|\mathcal{Y}^{v a l}-\hat{\mathcal{Y}}^{\text {val }}\right\|^{2}$ as the metric to select the best combinations of the tuning parameters. 


\section{Improve Model Local and Global Interpretability}

After the model is derived in Equation (5) and the transition and emission are defined in Equations (6) and (7), we will discuss how to improve the model interpretability by developing novel techniques for input variables identifications. Moreover, we aim to develop an interpretation module in this chapter to understand what happens exactly in the black-box model. In literature, there are two types of interpretability, global interpretability and local interpretability. Global interpretability refers to understand how the model makes decisions, based on a holistic view of its features and each of the learned components such as weights, other parameters, and structures, as defined in Chapter 2.3.2 in (Molnar, 2020). In the context of MMS, global interpretability refers to identify important input variables that are important for any output variables for all samples. We will discuss how to achieve global interpretability in the proposed DMMTL method in Section 4.1 .

Local interpretability refers to understand how the model makes decisions based on each individual sample. Local interpretability is quite important, given that the different output variables for different samples may depend on different sets of input variables, as defined in Chapter 2.3.4 in (Molnar, 2020). In the context of MMS, local interpretability refers to identify important input variables (e.g., process variables) for each output variable (e.g., quality index) for each individual sample (i.e., or a subset of samples). We will discuss how to achieve the local interpretability in the proposed DMMTL method in Section 4.2

\subsection{Improve Model Local Interpretability}

In this subsection, we will discuss how to achieve global model interpretability by identifying the input variables contributing to the prediction of the entire MMS systems by examining the model coefficients. In general, the $L_{2,1}$ penalty is able to pick up the most important input variables for each stage automatically and the non-zero value of the $L_{2}$ norm of different columns of $\mathbf{W}_{x k}$, or namely $\left\|\mathbf{w}_{i, x k}\right\|_{2}$, correspond to the most important input variables in stage $k$. These important input variables are selected because they contribute significantly to the entire MMS systems (i.e., any output variable in the future stages). We will then discuss how to achieve local interpretation by identifying important sensing variables with respect to each individual output sensor (i.e., diagnostics) for a single sample.

\subsection{Improve Model Global Interpretability}

In this subsection, we will discuss how to achieve local model interpretability by identifying the important input variables that relate the most to one specific quality index for any particular subset of samples. Motivated by (Apley, 2016), we propose the gradient tracking technique to achieve the input variable selection 
for a selected output variable for a particular sample for local interpretability. This is done through tracking back the gradient of the identified output variable $y_{k j}$ according to each individual input variable by backpropagation through the output linkage function $y_{k j}=g_{k j}\left(\mathbf{h}_{k} ; \theta_{k}^{g}\right)$ and the state transition matrix $\mathbf{h}_{k}=$ $f_{k}\left(\mathbf{h}_{k-1}, \mathbf{x}_{k}, \mathbf{y}_{k-1} ; \theta_{k}^{h}\right)$. If we choose a linear function for both $g_{k j}(\cdot)$ and $f_{k}(\cdot)$, the relationship between $y_{k j}$ and each individual $x_{k}$ is also linear. However, for a nonlinear output function and state transition matrix, the exact functional form of each $y_{k j}$ and input variable $\mathbf{x}$ can be quite complex. To analyze the relationship between the input and output variables, we propose to use the Taylor expansion of $y_{k j}$ according to the input variable $\mathbf{x}$ as follows:

$$
y_{p q}(\mathbf{x}+\Delta \mathbf{x})=\sum_{k=1}^{K} \sum_{i=1}^{n_{x k}} \frac{\partial y_{p q}}{\partial x_{k i}} \Delta x_{k i}+\sum_{k} \sum_{k^{\prime}} \sum_{i} \sum_{i^{\prime}} \Delta x_{k i} \frac{\partial^{2} y_{p q}}{\partial x_{k i} \partial x_{k^{\prime} i^{\prime}}} \Delta x_{k^{\prime} i^{\prime}}+O\left(\Delta x_{k i}^{3}\right)
$$

Therefore, the relative importance of a sensor can be computed by the gradient $\frac{\partial y_{p q}}{\partial x_{k i}}$. The first-order gradient information can be computed through the back-propagation through the sequential stages, as

$$
\frac{\partial y_{p q}}{\partial \mathbf{x}_{k}}=\frac{\partial y_{p q}}{\partial \mathbf{h}_{k^{\prime}}} \frac{\partial \mathbf{h}_{k^{\prime}}}{\partial \mathbf{h}_{k^{\prime}-1}} \cdots \frac{\partial \mathbf{h}_{k+1}}{\partial \mathbf{h}_{k}} \frac{\partial \mathbf{h}_{k}}{\partial \mathbf{x}_{k}}
$$

The detailed derivation for each component is shown in Appendix D. Because the gradient $\frac{\partial y_{p q}}{\partial \mathbf{x}_{k}}$ also depends on the value of $\mathbf{x}_{k}$, to obtain the relative importance of each input variable, we propose to compute the sum of squares of the gradient averaging over either the entire samples as $\frac{1}{N_{n}} \sum_{n=1}^{N_{n}}\left(\frac{\partial y_{p q}}{\partial \mathbf{x}_{k}^{n}}\right)^{2}$ or a selected number of defective samples for local interpretation. It is worth noting that in many applications, the important sensors may not be consistent across different ranges of $\mathbf{x}_{k}$. In this case, it may be useful to divide the entire region of $\mathbf{x}_{k}$ into different windows and compute this average for each window.

\section{Simulation}

In this section, we simulate a multistage manufacturing process with 9 stages. For the $k^{\text {th }}$ stage there are $n_{x}=90$ input variables, denoted as $\mathbf{x}_{k}=\left(x_{k, 1}, \cdots, x_{k, n_{x}}\right)$ and $n_{y}=6$ output variables, denoted as $\mathbf{y}_{k}=\left(y_{k, 1}, \cdots, y_{k, n_{y}}\right)$. For the input variable we simulate $x_{k, i} \stackrel{i . i . d}{\sim} N(0,1)$. We will discuss how to generate the output variables in three different scenarios. In all simulation scenarios, we assume there is a hidden state representation $\mathbf{h}_{k}$. We will assume three different hidden state $\mathbf{h}_{k}$ transition scenarios.

Case 1. One Unified MMS In this case, we generate the MMS with linear hidden state transition model as $\mathbf{h}_{k}=\mathbf{W}_{x k} \mathbf{x}_{k}+\mathbf{U}_{h k} \mathbf{h}_{k-1}+\mathbf{b}_{k}$. Furthermore, for each output variable $y_{k, i}$, it is generated from the $\mathbf{y}_{k}=\mathbf{W}_{y k} \mathbf{h}_{k}+\boldsymbol{\epsilon}_{k i}$, where $\epsilon_{k i} \sim N\left(0, \sigma^{2}\right)$ and $\sigma=0.5$. Each element of $\mathbf{W}_{x k}$ is generated from 
normal distribution $N\left(0, \frac{1}{\sqrt{n_{x}}}\right)$. Each element of $\mathbf{b}_{k}, \mathbf{U}_{h k}, \mathbf{W}_{y k}$ is generated from $N\left(0, \frac{1}{\sqrt{n_{h}}}\right)$, where $n_{h}$ is the dimensionality of $\mathbf{h}_{k}$. Furthermore, for each stage $k$, we will generate 15 out of $n_{x}=90$ as unimportant sensors for each stage by setting the last 15 rows of $\mathbf{W}_{x k}$ to be 0 .

Case 2. Three Parallel Sensor Groups in one MMS In this case, we assume that the input variable $\mathbf{x}_{k}$ and output variable $\mathbf{y}_{k}$ can be divided into three groups as $\mathbf{x}_{k}=\left\{\mathbf{x}_{k}^{(g)}, g=1,2,3\right\}$ and $\mathbf{y}_{k}=\left\{\mathbf{y}_{k}^{(g)}, g=1,2,3\right\}$. For each group, we generate $n_{x}^{(g)}=30$ input variables and $n_{y}^{(g)}=2$ output variables. For each group $g$, the hidden state $\mathbf{h}_{k}^{(g)}$ follows its own transition and only relates to the corresponding output variables $\mathbf{y}_{k}^{(g)}$ as $\mathbf{h}_{k}^{(g)}=\mathbf{W}_{x k}^{(g)} \mathbf{x}_{k}^{(g)}+\mathbf{U}_{h k}^{(g)} \mathbf{h}_{k-1}^{(g)}+\mathbf{b}_{k}^{(g)}$ and $\mathbf{y}_{k}^{(g)}=\mathbf{W}_{y k}^{(g)} \mathbf{h}_{k}^{(g)}+\boldsymbol{\epsilon}_{k i}^{(g)}, g=1,2,3$. In this case, each element of $\mathbf{W}_{y k}^{(g)}$, $\mathbf{b}_{k}^{(g)}$, and $\mathbf{U}_{h k}^{(g)}$ is generated from the normal distribution $N\left(0, \frac{1}{\sqrt{n_{h}^{(g)}}}\right)$, where $n_{h}^{(g)}$ is the dimensionality of the hidden state $\mathbf{h}_{k}^{(g)}$. Each element of $\mathbf{W}_{y k}^{(g)}$ is generated from $N\left(0, \frac{1}{\sqrt{n_{x}^{(g)}}}\right)$ and $\epsilon_{k i} \sim N\left(0, \sigma^{2}\right)$. Furthermore, for each stage $k$ and group $g$, we generate 5 unimportant input variables for each stage by setting the last 5 rows of $\mathbf{W}_{x k}^{(g)}$ to be 0 .

Case 3. Three Manufacturing Lines in one MMS In this case, we assume that the state transition is only related to the hidden state that is three-stage apart $\mathbf{h}_{k}=\mathbf{W}_{x k} \mathbf{x}_{k}+\mathbf{U}_{h k} \mathbf{h}_{k-3}+\mathbf{b}_{k}$. In other words, the three groups of output variables in the stage $k=1,4,7, k=2,5,8, k=3,6,9$ are correlated within the group but independent from each other. However, this relationship is assumed to be unknown. We generate $n_{x}=90$ input variables and $n_{y}=6$ output variables in each stage. Each element of $\mathbf{W}_{x k}$ is generated from normal distribution $N\left(0, \frac{1}{\sqrt{n_{x}}}\right)$. Each element of $\mathbf{b}_{k}, \mathbf{U}_{h k}, \mathbf{W}_{y k}$ is generated from $N\left(0, \frac{1}{\sqrt{n_{h}}}\right)$, where $n_{h}$ is the dimensionality of $\mathbf{h}_{k}$. Furthermore, for each stage $k$, we generate 15 unimportant input variables for each stage by setting the last 15 rows of $\mathbf{W}_{x k}$ to be 0 .

In all cases, we assume that the relationship between different stages and different sensing variables are not known. The goal is to predict each output variable $y_{k j}$, given the input variables up to stage $k$, denoted by $\mathbf{x}_{1}, \cdots, \mathbf{x}_{k}$ without relying on the specific relationship between stages. Finally, we divide the data into training $\left(\mathbf{x}^{t r}, \mathbf{y}^{t r}\right)$ and testing $\left(\mathbf{x}^{t e}, \mathbf{y}^{t e}\right)$ and use the relative mean of squared error (RMSE) $\sum_{k} \sum_{j}\left\|y_{k, j}^{t e}-\hat{y}_{k, j}^{t e}\right\|^{2} /\left\|y_{k, j}^{t e}-\bar{y}_{k, j}^{t r}\right\|^{2}$ for performance evaluation. If RMSE is smaller than 1 it means that the estimator is able to get some signals by beating the naive predictor as the training mean. A smaller RMSE indicates better performance. For benchmark methods, we will compare the proposed MMS model framework with other prediction methods that focus on modeling each single input variable individually. The modeling methods we are comparing are linear regression (LR), elastic net (EN), random forest (RF) and multi-layer perceptron (MLP). We will also include the stream of variation (SoV) as an oracle method since it assumes the true transition and output function is known. For the MLP, we build a 2-layer fully connected 
Table 2: Prediction RMSE and standard deviation

\begin{tabular}{|c|c|c|c|}
\hline & \multicolumn{3}{|c|}{ RMSE } \\
\hline & Case 1 & Case 2 & Case 3 \\
\hline \hline DMMTL & $\mathbf{0 . 0 9 0 ( \mathbf { 0 . 0 3 7 } )}$ & $\mathbf{0 . 1 3 8 ( \mathbf { 0 . 0 6 0 } )}$ & $\mathbf{0 . 1 3 4}(\mathbf{0 . 0 5 7})$ \\
\hline MEN & $0.239(0.134)$ & $0.192(0.108)$ & $0.166(0.072)$ \\
\hline LR & $0.577(0.632)$ & $0.666(1.173)$ & $1.213(1.653)$ \\
\hline EN & $0.273(0.162)$ & $0.150(0.090)$ & $0.273(0.127)$ \\
\hline RF & $0.863(0.079)$ & $0.768(0.123)$ & $0.822(0.085)$ \\
\hline MLP & $0.728(0.266)$ & $0.808(0.330)$ & $1.006(0.413)$ \\
\hline
\end{tabular}

neural network to link the input variables up to stage $k$ to each output variable $y_{k j}$. We also compare with another Multi-task learning method, namely the Multi-task Elastic Net (MEN), which combines the MTL to model the multivariate response in each stage $\mathbf{y}_{k}$ and elastic net for variable selection. MEN assumes that the same variables should be selected for different tasks/sensors in each stage. For more details about MEN methods, please check the Appendix . To be fair, we will look at the supervised learning models to predict the output sensors in the last stage and look at the magnitude of the model coefficients for each sensor. The results are shown in Table 7

From Table 7. we see that DMMTL is able to obtain the smallest RMSE in all cases compared to other benchmark methods. This superiority is due to the following three reasons: 1) Benefit of modeling the multiple output variables jointly. MEN models all output variables in each stage jointly by assuming that these model coefficients share the same sparsity structure, which can often lead to better modeling accuracy. This benefit can be seen in Case 3 by comparing the RMSE of MEN, about 0.166, with the RMSE of EN, about 0.273 . However, we have to mention that in the case when the output variables are not correlated, this could lead to a worse result. For example, in Case 2, there are 3 different variable groups in each manufacturing stage and EN achieves the RMSE of 0.150, which is better than MEN with the RMSE as 0.192). 2) Benefit of jointly modeling the manufacturing stages. MEN models do not model the output variables in all stages jointly like DMMTL. In all cases, DMMTL achieves a smaller RMSE than that of MEN. For example, in Case 1, DMMTL achieves an RMSE of 0.090, which is smaller than the second best, namely the MEN, which achieves RMSE of 0.239. This shows the advantage of modeling all output variables jointly in all stages. 3) Benefit of sparsity penalty for feature selection. This benefit can be seen clearly by comparing the EN with LR and MLP. Without any sparsity constraint, both MLP and LR perform badly with a much larger variance due to the model over-fitting. In comparison, EN clearly outperforms these two methods in all cases.

To understand how the model works, we also plot the RMSE of the top 3 methods, namely EN, MEN and DMMTL in Figure 3. We can conclude that in Case 1, DMMTL is able to keep a consistent RMSE over 


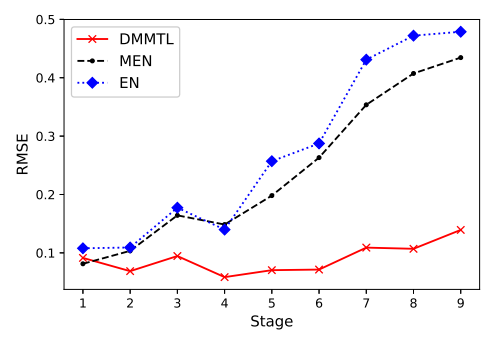

(a) Case 1

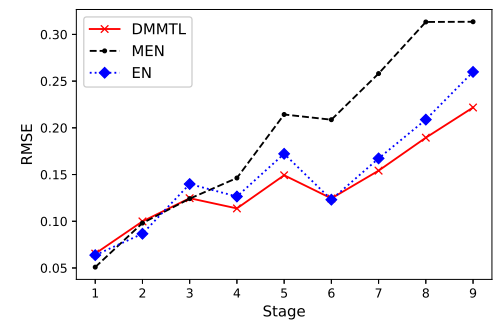

(b) Case 2

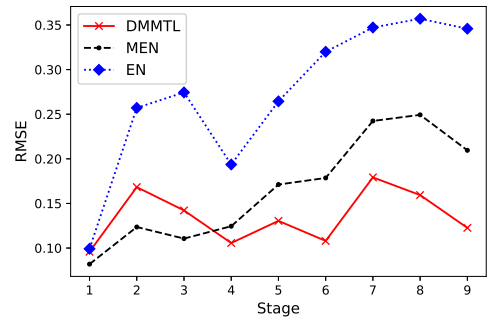

(c) Case 3

Figure 3: RMSE according to each stage

all manufacturing stages due to the information provided by the output variables in the intermediate stages. However, EN and MEN normally demonstrate an increasing trend of the RMSE over stages. The reason for this is that for later stages, the number of input variables increases dramatically. Without the guidance of the output variables in the intermediate stages, both EN and MEN cannot find the important variables easily. In Case 2, the RMSEs of all methods increase over the stages due to the decrease in dependency between the manufacturing stages. However, DMMTL still outperforms others. In Case 3, DMMTL has a slightly larger error compared to MEN in the initial stages. The reason for this is that the first three stages in Case 3 are actually completely independent. However, DMMTL is forced to learn a dependency between these stages, which could lead to a worse result. When the stages become dependent after Stage 3 (e.g., stage 4 is related to stage 1 , and stage 5 is related to stage 2), DMMTL is able to quickly exploit this dependency and outperform all other benchmark methods.

Furthermore, we would like to compare the input variable selection accuracy for Case 2 and Case 3 for the last output variable in the last stage (Stage 9). It is worth noting that only the proposed method, EN and MEN, are able to perform the feature selection by selecting the non-zero elements of the model due to the sparsity penalty. Other benchmark models, such as LR, RF, and MLP cannot perform variable selection naively. Recall that the data are already normalized to mean 0 and variance 1 . For LR, we will use the absolute value of the model coefficient directly for the variable importance score. For MLP, we will use only the norm of the model coefficient of the first layer (connected to the input variables) as the input variable importance score. For RF, we propose to use the feature importance metric computed by the average accuracy gain of each split according to each individual variable. The result of the percentage of the input variable identification is shown in Table 3.

To evaluate the variable selection accuracy, we can view the input variable identification problem as the classification problem and we also compute the precision, recall, and AUC score in Table 3 . Precision is defined as the percentage of identified variables that are actually important. Recall is defined as the 
Table 3: Input Variable Identification Accuracy

\begin{tabular}{|c|cc|cc|cc|}
\hline & \multicolumn{2}{|c|}{ Precision } & \multicolumn{2}{c|}{ Recall } & \multicolumn{2}{c|}{ AUC } \\
\hline & Case 2 & Case 3 & Case 2 & Case 3 & Case 2 & Case 3 \\
\hline DMMTL & $\mathbf{0 . 7 9 5}$ & $\mathbf{0 . 8 6 7}$ & $\mathbf{0 . 5 1 5}$ & $\mathbf{0 . 8 7 1}$ & $\mathbf{0 . 8 1 0}$ & $\mathbf{0 . 9 5 8}$ \\
\hline MEN & 0.677 & 0.834 & 0.280 & 0.671 & 0.633 & 0.916 \\
\hline LR & 0.143 & 0.724 & 0.022 & 0.3511 & 0.486 & 0.689 \\
\hline EN & 0.189 & 0.752 & 0.031 & 0.404 & 0.465 & 0.706 \\
\hline RF & 0.167 & 0.589 & 0.027 & 0.191 & 0.463 & 0.599 \\
\hline MLP & 0.333 & 0.348 & 0.071 & 0.071 & 0.503 & 0.526 \\
\hline
\end{tabular}

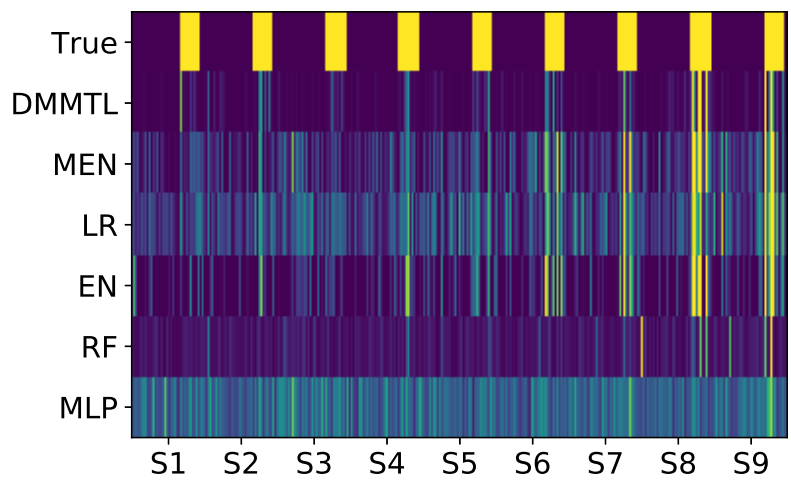

(a) Case 2

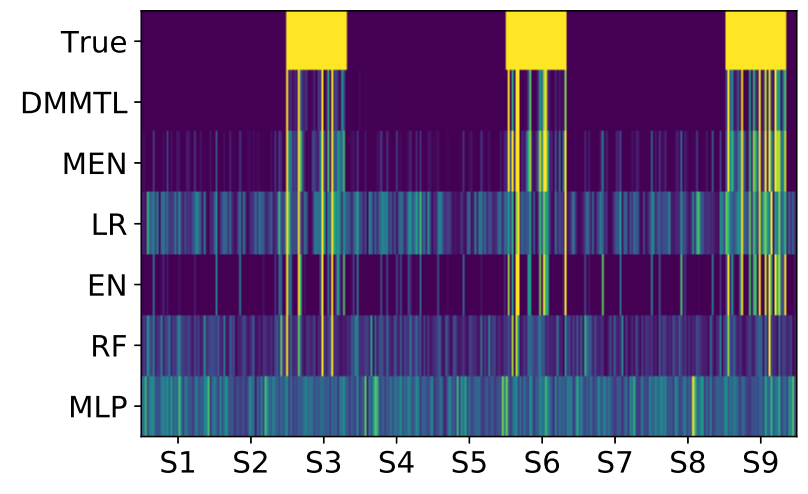

(b) Case 3

Figure 4: Identified Important Sensor for Case 2 and Case 3

percentage of important variables that are actually identified. AUC is defined as the area under the receiver operating characteristic curve. The threshold to determine the important input variable is set to maintain the false positive rate as 5\%. From Table 3, we can conclude that DMMTL is able to accurately identify the input variables compared to other benchmark methods in both Case 2 and 3 with the highest precision, recall, and AUC score. MEN performs the second best, due to the ability to use information from multiple output sensors jointly within each stage. MEN is especially able to achieve a much higher recall score than the other benchmark methods, showing the strength of a multi-task learning framework. To better understand how each method performs feature selection, we also plot the feature importance score computed by each method in Figure 4. From Figure 4, we can conclude that DMMTL is able to use the least number of input variables to achieve the best prediction power compared to all other benchmark methods due to the group lasso penalty.

\section{Case Study}

In this case study, we apply DMMTL to model the diaper assembly process introduced in Section 1 . We divide the whole converting process into five stages and identify 484 process variables (e.g. temperature, 
Table 4: Number of Input and Output Variables for Each Stage

\begin{tabular}{|c|c|c|c|c|c|}
\hline & Stage 1 & Stage 2 & Stage 3 & Stage 4 & Stage 5 \\
\hline \hline Input Variables & 110 & 86 & 165 & 120 & 0 \\
\hline Output Variables & 20 & 64 & 10 & 90 & 16 \\
\hline
\end{tabular}

pressure, etc.) as inputs and 200 quality measurements (e.g. product dimensions) as output variables in the model. Due to the complex physical process involved, it is very hard to derive the physics relationship between the input variables and output variables. The detailed information of the number of input and output sensors in each stage is shown in Table 4 . Due to the privacy constraint, the name of the stages and the name of the sensors can not be given here. Furthermore, to increase the prediction power of our model, we also use the output measurements from the previous stage as input variables to the next stage. Because the manufacturing data is very noisy, we use both the Huber loss function and the traditional residual sum of squares for comparison. We also compare DMMTL with several benchmark methods, including the multi-task elastic net (MEN), ridge regression (RR), elastic net $(\mathrm{EN})$, random forest $(\mathrm{RF})$, and multilayer perception (MLP). We do not include linear regression (LR) in the comparison because its parameter estimation is numerically unstable and it can also be seen as a special case of RR without adding penalties.

One interesting phenomenon in the realistic case study is that not all output variables can be predicted well based on the input variables. Given the complexity of the manufacturing process, even with 484 input variables some of the important characteristics of the underlying process are still not measured by the sensors. Therefore, we try to find a model that can achieve excellent predictive power for most of the output variables. Furthermore, even knowing which output variables cannot be predicted is useful information. This could guide adding more sensors or increasing sample frequency. Finally, for more complicated cases, we used two-layer neural networks for both emission and transition functions.

To compare how the methods are able to identify related output variables, we first compute the RMSE of all 200 output variables. Recall that the RMSE is defined as $\sum_{k} \sum_{j}\left\|y_{k, j}^{t e}-\hat{y}_{k, j}^{t e}\right\|^{2} /\left\|y_{k, j}^{t e}-\bar{y}_{k, j}^{t r}\right\|^{2}$ and if its value is smaller than 1 then that indicates the model can achieve a better prediction than the naive predictor based on the mean response value. To evaluate the performance of different methods for identifying the important variables, we first compute the $20 \%, 40 \%, 50 \%$ and $70 \%$ quantiles of the 200 RMSE scores in Table 5 for 200 output variables for each method. We then choose thresholds on the RMSE ranging from 0.05 to 0.95 and define the number of output variables with the RMSE score smaller than the threshold as the number of identified related output variables. In Figure 5 , we plot the number of identified related output variables for different thresholds.

Table 5 shows that DMMTL can achieve a lower RMSE over all quantiles $20 \%, 40 \%, 50 \%$ and $70 \%$. In 
Table 5: Quantiles of Prediction RMSE

\begin{tabular}{|c|c|c|c|c|}
\hline Quantile & $20 \%$ & $40 \%$ & $50 \%$ & $70 \%$ \\
\hline \hline DMMTL (Huber) & $\mathbf{0 . 2 9}$ & $\mathbf{0 . 7 3}$ & 0.87 & 1.01 \\
\hline DMMTL (MSE) & 0.39 & 0.71 & $\mathbf{0 . 8 1}$ & $\mathbf{0 . 9 9}$ \\
\hline Multi-task Elastic Net & 0.79 & 0.88 & 0.92 & 0.99 \\
\hline Elastic Net & 0.60 & 0.91 & 0.99 & 1.00 \\
\hline Random Forest & 0.53 & 0.79 & 0.93 & 1.11 \\
\hline Multi-layer Perception & 0.76 & 1.83 & 2.59 & 5.68 \\
\hline
\end{tabular}

particular, for the 20\% quantile, DMMTL with Huber and mean square error loss function achieve the RMSE of 0.29 and 0.39 , which indicates the strong prediction power (i.e., much smaller than 1). Furthermore, when comparing the median of the RMSE, only the proposed methods are able to achieve RMSE lower than 0.9. This can also be seen from Figure 5 that DMMTL outperforms all other benchmark methods due to the ability to combine the modeling with multiple output variables in different stages in a unified model. Furthermore, from Table 5, we see that DMMTL with the Huber loss function is able to outperform the mean square error loss function at the $20 \%$ and $40 \%$ quantiles or when the threshold is small as shown in Figure 5. The reason is that the Huber loss function is more robust to outlier sensing variables and therefore, will focus more on reducing the loss functions of the output variables which are truly correlated to the input variables. RF and EN's performances follow immediately after our proposed method because they have the ability to select important variables. MLP, in general, performs worse due to the lack of penalization and feature selection. MEN performs the worst in this example since there are many uncorrelated output variables even in the same stage, which violates the assumption of MEN that the sparsity structure for all input variables in the same stage is the same.

To demonstrate the performance of the prediction accuracy for all methods, in Figure 6 we plot the predicted signals and the true signal for the output variables for both training and testing data. A dashed black line is added in each plot to separate the training and testing data. We select output sensors 16, 33, 75, 132, 197 for demonstration in stage 1, 2, 3, 4, 5, respectively. The RMSE according to these output sensors and the selected number of input variables of each method are shown in Table 6 and Table 7 .

From Figure 6 and Table 6, we first conclude that these output variables share similar patterns. For example, Output 16 has a meanshift during time 1961 and Output 33, 75, and 197 has a meanshift at time 2813. Output 132 have meanshifts at both time points. DMMTL is able to achieve the least RMSE among all methods due to its ability to combine all output sensors in a unified model, therefore leading to a better model for all output sensors. EN also achieves good performance for Output 16 and 75 . For Output 33 and 132, only DMMTL is able to accurately predict the trend. RF sometimes does not capture the trend correctly. MLP typically overfits the data, and therefore it normally produces much larger noises in the 


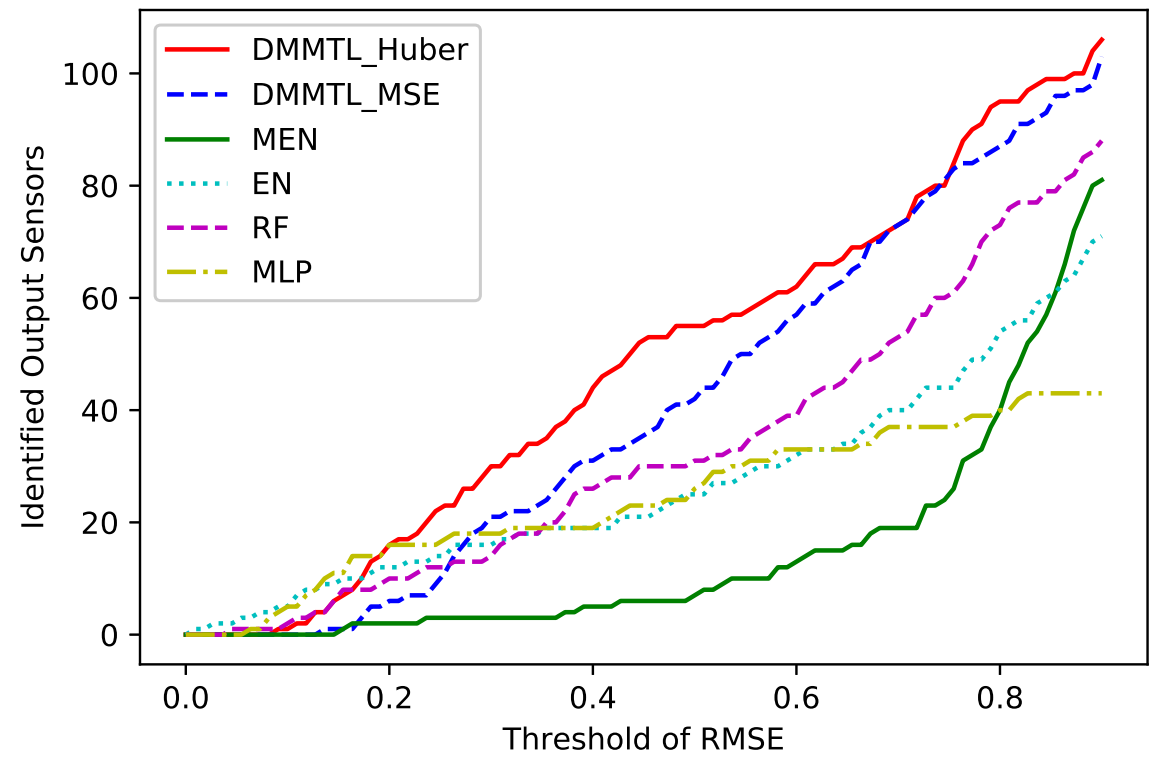

Figure 5: Number of identified important output variables with different threshold on the prediction RMSE

Table 6: Number of Input Sensors Identified

\begin{tabular}{|c|ccccc|}
\hline Sensor \# & 16 & 33 & 75 & 132 & 197 \\
\hline DMMTL & 16 & 20 & 21 & 21 & 21 \\
\hline MEN & 35 & 35 & 35 & 35 & 36 \\
\hline EN & 18 & 18 & 18 & 16 & 16 \\
\hline RF & 21 & 116 & 151 & 30 & 342 \\
\hline MLP & 119 & 225 & 225 & 593 & 682 \\
\hline
\end{tabular}

testing data. MEN typically underfits the data due to its strong assumption that the models for output sensors in the same stage must share the same sparsity patterns. In terms of the number of input sensors identified, typically EN is able to identify the least number of input sensors, followed by DMMTL and MEN. MLP is not able to perform feature selection, which leads to severe over-fitting. In conclusion, we see that DMMTL is able to achieve the least RMSE with a relatively small number of selected input sensors.

Finally, in Figure 7, we also plot the top three important input variables identified for all these five output variables. Among them, Input 744 can explain the meanshift at time 2813 for all output variables and Input

Table 7: RMSE

\begin{tabular}{|c|ccccc|}
\hline Sensor \# & 16 & 33 & 75 & 132 & 197 \\
\hline DMMTL & $\mathbf{0 . 1 2}$ & $\mathbf{0 . 0 7}$ & $\mathbf{0 . 0 5}$ & $\mathbf{0 . 3 7}$ & $\mathbf{0 . 7 3}$ \\
\hline MEN & 0.59 & 0.76 & 0.83 & 1.21 & 0.95 \\
\hline EN & 1.00 & 0.89 & 0.09 & 1.00 & 0.98 \\
\hline RF & 0.39 & 0.65 & 0.41 & 7.73 & 0.83 \\
\hline MLP & 0.16 & 3.94 & 0.32 & 2.84 & 7.33 \\
\hline
\end{tabular}




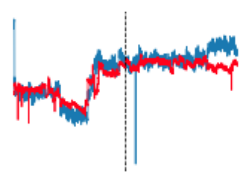

(a) DMMTL

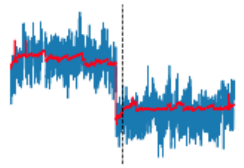

(f) DMMTL

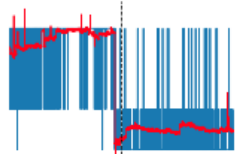

(k) DMMTL

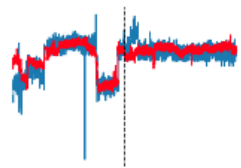

(p) DMMTL

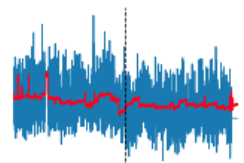

(u) DMMTL

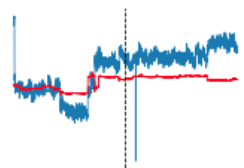

(b) MEN

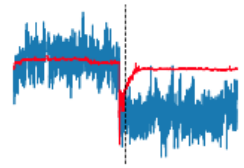

(g) MEN

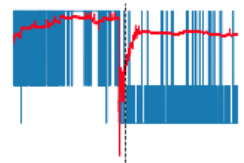

(l) MEN

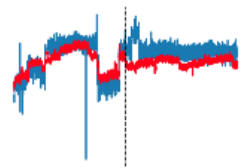

(q) MEN

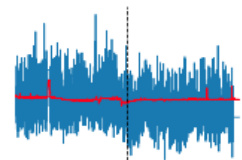

(v) MEN

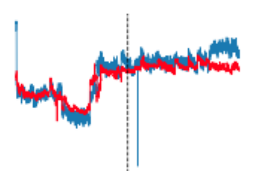

(c) $\mathrm{EN}$

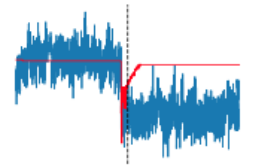

(h) EN

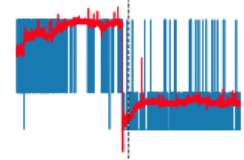

(m) EN

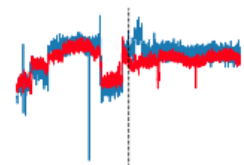

(r) EN

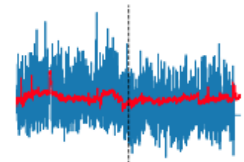

(w) EN

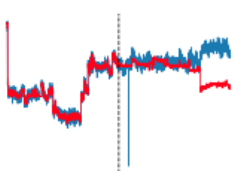

(d) $\mathrm{RF}$

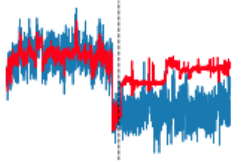

(i) $\mathrm{RF}$

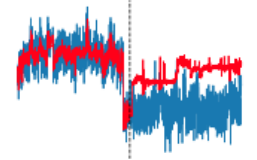

(n) $\mathrm{RF}$

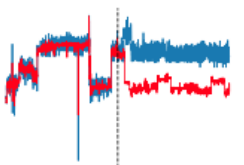

(s) RF

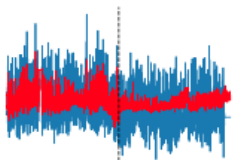

(x) RF

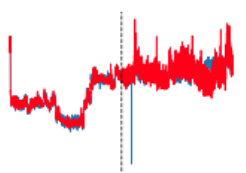

(e) MLP

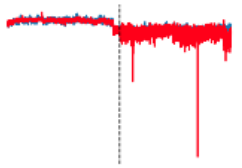

(j) MLP

Figure 6: Example of Sensor Prediction (1st row: sensor 16, 2nd row: sensor 33, 3rd row: sensor 75, 4th row: sensor 132, 5th row: sensor 197) 


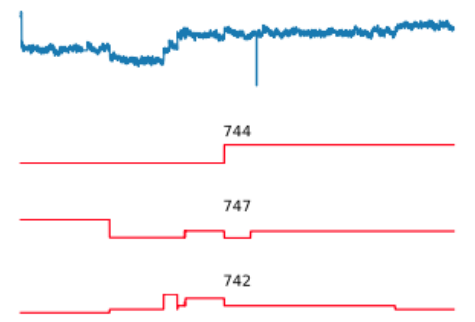

(a) Output 16

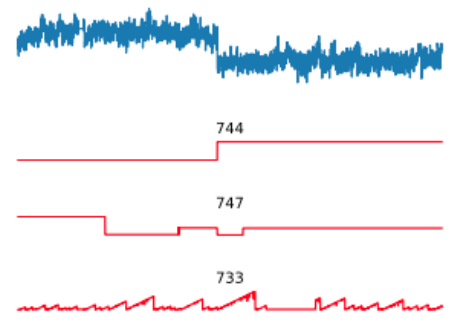

(b) Output 33
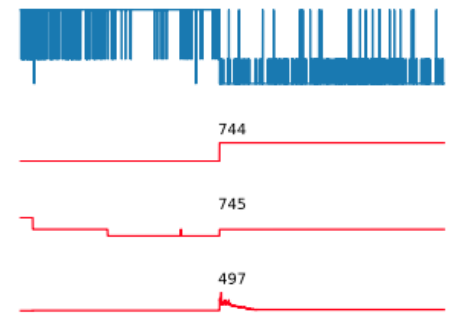

(c) Output 75

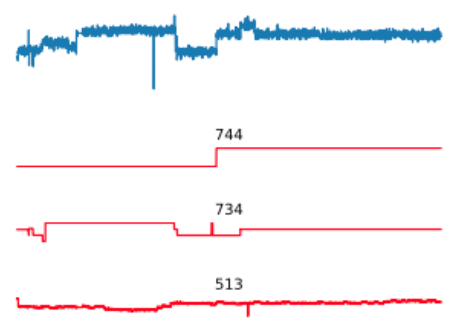

(d) Output 132

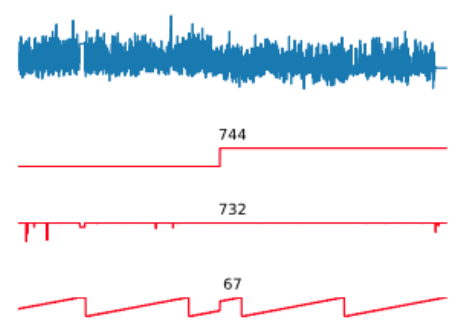

(e) Output 197

Figure 7: Top three important input variables identified by DMMTL for Output 16, 33, 75, 132 and 197

747 can explain some of the other small meanshifts for Output 16 and 33. Finally, Input 733 and Input 67 are able to explain the small increasing trend in Output 33 and 197, respectively. We have validated that the selected input variables indeed can explain the variation for the selected output variable from the domain knowledge.

\section{Conclusion}

Modeling complex multistage manufacturing systems is an important research topic for accurate process prediction, monitoring, diagnosis and control. This paper proposes a deep transition model with multitask learning to jointly model all output sensing variables with the input sensing variables according to the sequential production line structure. Furthermore, since the dimensionality of the input sensing variables and output sensing variables can be very high, we suggest reducing the dimensionality by utilizing the sparse regularization and robust Huber loss function to select the important sensing variables. DMMTL has been tested through several simulated studies and a realistic case study of a real diaper manufacturing system. The results demonstrate that it achieves a better prediction accuracy as well as a better local and global interpretability by identifying important relationship between input and output sensing variables.

There are several future research directions that we would like to investigate. One is to extend this method to heterogeneous measurements with more stage dependencies (e.g., tree structures) other than the 
production line set up in series. Another extension is to study the proposed algorithm under the stochastic transition of the hidden variables similar to the stream of variation models.

\section{A Relationship to Stream of Variation}

The foundation of SoV methodology is a mathematical model that links the output variables (e.g., key quality characteristics of the product ) with key input variables (e.g., key process sensing variables) through the state space representation.

$$
\mathbf{h}_{k}=\mathbf{W}_{x k} \mathbf{x}_{k}+\mathbf{U}_{h k} \mathbf{h}_{k-1}+\mathbf{e}_{h k}, \mathbf{y}_{k}=\mathbf{V}_{y k} \mathbf{h}_{k}+\mathbf{e}_{y k}
$$

The variable $\mathbf{h}_{k}$ is the state vector representing the output variables at stage $k$. $\mathbf{e}_{h k}$ and $\mathbf{e}_{y k}$ are the modeling error and measurement error, respectively. The coefficient matrices $\mathbf{W}_{x k}, \mathbf{U}_{h k}$, and $\mathbf{V}_{y k}$ are determined by product and process design information at stage $k . \mathbf{W}_{x k}$ represents the impact of the new stage process to the product. $\mathbf{U}_{h k}$ represents the transition from stage $k-1$ to stage $k . \mathbf{V}_{y k}$ is the measurement matrix, which links the hidden state $\mathbf{h}_{k}$ and the output $\mathbf{y}_{k}$.

These mathematical models have achieved great success in MMS modeling by integrating the product and process design information and modeling the variation propagation in the MMS. However, the SoV methodology assumes that the key output variables $\mathbf{y}_{k}$ and key input variables $\mathbf{x}_{k}$ have been correctly identified. Furthermore, it requires the matrices $\mathbf{W}_{x k}, \mathbf{U}_{h k}$, and $\mathbf{V}_{y k}$ are known in each stage $k$, which is not possible if the system is too complex. Finally, it assumes the linear transition matrix between states which could be an over-simplification in many real cases. However, SoV assumes that the transition between the state variables are known as the linear stochastic function. The proposed DMMTL assumes that the transition is unknown nonlinear functions. Extending the framework to stochastic functions would be one of our future work.

\section{B Relationship to Recurrent Neural Network}

The formulation of RNN methodology is a neural network that links the output variables $\mathbf{y}_{k}$, input variables $\mathbf{x}_{k}$ in a neural network. More specifically, at each time $k$, the output $\mathbf{y}_{k}$ and input $\mathbf{x}_{k}$ are linked together via the hidden state $\mathbf{h}_{k}$ in (Equation 12 ).

$$
\mathbf{h}_{k}=\sigma\left(\mathbf{W}_{x} \mathbf{x}_{k}+\mathbf{U}_{h} \mathbf{h}_{k-1}+\mathbf{b}_{h}\right), \mathbf{y}_{k}=\mathbf{V}_{y} \mathbf{h}_{k}+\mathbf{b}_{y}
$$


In RNN, it is typically assumed that the system is time-invariant, which means the model parameters $\mathbf{W}_{x}, \mathbf{U}_{x}, \mathbf{V}_{y}$ are independent of $k$. RNN has achieved great success in machine translation problems (Graves et al. 2013a), handwriting recognition (Graves and Schmidhuber, 2009) and speech recognition (Graves et al. 2013b). Furthermore, model parameters $\mathbf{W}_{x}, \mathbf{U}_{x}, \mathbf{V}_{y}$ can be learned in an end-to-end fashion via the combination of back-propagation (LeCun et al. 1990) and stochastic gradient descent (Bottou, 2010).

However, the major limitation of using RNN in MMS is that different manufacturing stages are inherently different. The underlying physics is entirely different for each stage which not only results in the different transition matrix $\mathbf{W}_{x}, \mathbf{U}_{h}$, and $\mathbf{V}_{y}$. RNN also assumes the same set of variables are predicted in each time. However, in MMS, different quality inspection sensors are set up in each manufacturing stage. Finally, RNN is a complicated model and can not achieve input and output variable selection as the proposed approach.

\section{Proof of Proposition 2:}

Proof. Considering the loss function

$$
\min _{a_{k j}} \sum_{k, j}\left\|y_{k j}-g_{k j}\left(\mathbf{h}_{k} ; \boldsymbol{\Theta}\right)-a_{k j}\right\|^{2}+\lambda_{x} \sum_{i=1}^{n_{x, k}}\left\|\mathbf{w}_{i, x k}\right\|_{2}+\frac{\lambda}{2}\|\Theta\|^{2}+\gamma \sum_{k, j}\left\|a_{k j}\right\|_{1}
$$

Here, the loss function in 13 can be decoupled into each pair of $(k, j)$ individually. Therefore, each $a_{k j}$ can be solved individually by optimizing

$$
\left\|y_{k j}-g_{k j}\left(\mathbf{h}_{k} ; \boldsymbol{\Theta}\right)-a_{k j}\right\|^{2}+\gamma\left\|a_{k j}\right\|_{1}
$$

and can be solved by

$$
a_{k j}=S_{\gamma / 2}\left(y_{k j}-g_{k j}\left(\mathbf{h}_{k} ; \mathbf{\Theta}\right)\right)
$$

To optimize the $\mathbf{w}_{i, x k}$, we will follow the derivation of the proximal gradient algorithm the Taylor expansion of $\mathcal{L}(\Theta, \mathcal{A})$ as

$$
\mathcal{L}(\Theta, \mathcal{A}) \leq \mathcal{L}\left(\Theta^{(t-1)}, \mathcal{A}\right)+\sum_{i} \frac{\partial \mathcal{L}\left(\Theta^{(t-1)}, \mathcal{A}\right)}{\partial \mathbf{w}_{i, x k}}\left(\mathbf{w}_{i, x k}-\mathbf{w}_{i, x k}^{(t-1)}\right)+\frac{L}{2}\left\|\mathbf{w}_{i, x k}-\mathbf{w}_{i, x k}^{(t-1)}\right\|^{2}
$$


$L$ is the Lipschitz constance of $\mathcal{L}(\Theta, \mathcal{A})$. Therefore, we aim to minimize the upper bound of $\mathcal{L}(\Theta, \mathcal{A})+\mathcal{R}(\Theta, \mathcal{A})$ as follows:

$$
\begin{array}{r}
\min _{\left\{\mathbf{w}_{i, x k}\right\}} \mathcal{L}\left(\Theta^{(t-1)}, \mathcal{A}\right)+\sum_{i, k} \frac{\partial \mathcal{L}\left(\Theta^{(t-1)}, \mathcal{A}\right)}{\partial \mathbf{w}_{i, x k}}\left(\mathbf{w}_{i, x k}-\mathbf{w}_{i, x k}^{(t-1)}\right)+\frac{L}{2} \sum_{i, k}\left\|\mathbf{w}_{i, x k}-\mathbf{w}_{i, x k}^{(t-1)}\right\|^{2}+ \\
\lambda_{x} \sum_{i, k}\left\|\mathbf{w}_{i, x k}\right\|_{2}+\frac{\lambda}{2} \sum_{i, k}\left\|\mathbf{w}_{i, x k}\right\|^{2}
\end{array}
$$

Here, we find that the optimization can be decoupled to each individual $(i, k)$ as

$$
\min _{\mathbf{w}_{i, x k}}\left\|\mathbf{w}_{i, x k}-\frac{L}{L+\lambda}\left(\mathbf{w}_{i, x k}^{(t-1)}-\frac{1}{L} \frac{\partial \mathcal{L}\left(\Theta^{(t-1)}, \mathcal{A}\right)}{\partial \mathbf{w}_{i, x k}}\right)\right\|^{2}+\frac{2 \lambda_{x}}{L+\lambda} \sum_{i=1}^{n_{x, k}}\left\|\mathbf{w}_{i, x k}\right\|_{2}
$$

and can be solved in closed form as

$$
\mathbf{w}_{i, x k}^{(t)}=S_{\frac{\lambda_{x}}{L+\lambda}}\left(\frac{L}{L+\lambda}\left(\mathbf{w}_{i, x k}^{(t-1)}-\frac{1}{L} \frac{\partial \mathcal{L}\left(\Theta^{(t-1)}, \mathcal{A}\right)}{\partial \mathbf{w}_{i, x k}}\right)\right) .
$$

\section{Back-propagation along the Sequential Stages Over Parameters $\boldsymbol{\theta}_{k}$}

We will discuss how to efficiently optimize the model parameters $\Theta$ via stochastic gradient descent. Suppose we denote $\mathcal{X}^{n}, \mathcal{Y}^{n}$ as the $n^{\text {th }}$ sample of the entire dataset with $n=1, \cdots, N_{n}$, then the loss function can be decomposed as

$$
\mathcal{L}(\Theta ;\{\mathcal{X}\},\{\mathcal{Y}\})=\frac{1}{N_{n}} \sum_{n=1}^{N_{n}} \sum_{k=1}^{K} \sum_{j=1}^{N_{y, k}} \log P\left(y_{k j}^{n} \mid \mathbf{h}_{k} ; \Theta\right) .
$$

If the number of samples $N_{n}$ is large, averaging the gradient over the entire dataset is normally slow. To address this, we propose to apply the mini-batch stochastic gradient algorithm, which is widely used to optimize large-scale machine learning problems. In each iteration $t$, we can choose a subset of samples $\mathcal{N}_{t} \in\left\{1, \cdots, N_{n}\right\}$, where the gradient is only evaluated as the average of the subset of the entire samples in 16):

$$
\boldsymbol{\Theta}^{(t+1)}=\boldsymbol{\Theta}^{(t)}-\frac{1}{\left|\mathcal{N}_{t}\right|} \sum_{n \in \mathcal{N}_{t}} \frac{\partial \tilde{L}\left(\mathcal{X}^{n}, \mathcal{Y}^{n} ; \Theta^{(t)}\right)}{\partial \boldsymbol{\Theta}} .
$$

Now we will discuss how to compute the gradient according to the model coefficients $\boldsymbol{\Theta} . \boldsymbol{\Theta}=\left\{\boldsymbol{\theta}_{1}, \cdots \boldsymbol{\theta}_{K}\right\}$. First, the likelihood can be decomposed into different samples, stages, and output variables due to the 
conditional dependency of the hidden variables $\mathbf{h}_{k^{\prime}}$ in 17 .

$$
\frac{\partial}{\partial \boldsymbol{\theta}_{k}} \mathcal{L}(\mathbf{x}, \mathbf{y} ; \Theta)=\sum_{n=1}^{N_{n}} \sum_{k^{\prime}=1}^{K} \sum_{j=1}^{n_{y, k^{\prime}}} \frac{\partial}{\partial \boldsymbol{\theta}_{k}} \log P\left(y_{k^{\prime} j}^{n} \mid \mathbf{h}_{k^{\prime}} ; \boldsymbol{\theta}_{k}^{g}\right)
$$

Therefore, we need to compute $\frac{\partial}{\partial \boldsymbol{\theta}_{k}} \log P\left(y_{k^{\prime} j}^{n} \mid \mathbf{h}_{k^{\prime}} ; \boldsymbol{\theta}_{k}^{g}\right)$. For $k^{\prime}<k$, it is obvious that

$\frac{\partial}{\partial \boldsymbol{\theta}_{k}} \log P\left(y_{k^{\prime} j}^{n} \mid \mathbf{h}_{k^{\prime}} ; \boldsymbol{\theta}_{k}^{g}\right)=0$. However, if $k^{\prime} \geq k$, we can compute the gradient $\frac{\partial \log P\left(y_{k^{\prime}}^{n} \mid \mathbf{h}_{k^{\prime}} ; \boldsymbol{\theta}_{k}^{g}\right)}{\partial \boldsymbol{\theta}_{k}}$ recursively as $\frac{\partial \log P\left(y_{k^{\prime} j}^{n} \mid \mathbf{h}_{k^{\prime}} ; \boldsymbol{\theta}_{k}^{g}\right)}{\partial \boldsymbol{\theta}_{k}}=\frac{\partial \log P\left(y_{k^{\prime}}^{n} \mid \mathbf{h}_{k^{\prime}} ; \boldsymbol{\theta}_{k}^{g}\right)}{\partial \mathbf{h}_{k^{\prime}}} \frac{\partial \mathbf{h}_{k^{\prime}}}{\partial \mathbf{h}_{k^{\prime}-1}} \cdots \frac{\partial \mathbf{h}_{k+1}}{\partial \mathbf{h}_{k}} \frac{\partial \mathbf{h}_{k}}{\partial \boldsymbol{\theta}_{k}}$. By plugging in this into 17, we can derive the gradient according to the state transition parameters 18 .

$$
\frac{\partial}{\partial \boldsymbol{\theta}_{k}^{h}} \mathcal{L}(\mathbf{x}, \mathbf{y} ; \Theta)=\sum_{k^{\prime}=k}^{K} \sum_{j=1}^{n_{y, k^{\prime}}} \frac{\partial \log P\left(y_{k^{\prime} j}^{n} \mid \mathbf{h}_{k^{\prime}} ; \boldsymbol{\theta}_{k}^{g}\right)}{\partial \mathbf{h}_{k^{\prime}}} \frac{\partial \mathbf{h}_{k^{\prime}}}{\partial \mathbf{h}_{k^{\prime}-1}} \cdots \frac{\partial \mathbf{h}_{k+1}}{\partial \mathbf{h}_{k}} \frac{\partial \mathbf{h}_{k}}{\partial \boldsymbol{\theta}_{k}^{h}}
$$

Finally, to derive the gradient according to output coefficient $\boldsymbol{\theta}_{k}^{g}$, we can derive

$$
\frac{\partial}{\partial \boldsymbol{\theta}_{k}^{g}} \mathcal{L}(\mathbf{x}, \mathbf{y} ; \Theta)=\sum_{j=1}^{n_{y, k}} \frac{\partial \log P\left(y_{k j}^{n} \mid \mathbf{h}_{k} ; \boldsymbol{\theta}_{k}^{g}\right)}{\partial \boldsymbol{\theta}_{k}^{g}} .
$$

We would then discuss how to compute the gradient based for $\frac{\partial \log P\left(y_{k j} \mid \mathbf{h}_{k} ; \boldsymbol{\theta}_{k}^{g}\right)}{\partial \mathbf{h}_{k}}, \frac{\partial \log P\left(y_{k j} \mid \mathbf{h}_{k} ; \boldsymbol{\theta}_{k}^{g}\right)}{\partial \boldsymbol{\theta}_{k}^{g}}, \frac{\partial \mathbf{h}_{k}}{\partial \boldsymbol{\theta}_{k}^{h}}$, $\frac{\partial \mathbf{h}_{k}}{\partial \mathbf{h}_{k-1}}$. The computation is shown as follows:

- Emission layer

$$
\begin{aligned}
& \frac{\partial \log P\left(y_{k j} \mid \mathbf{h}_{k} ; \boldsymbol{\theta}_{k}^{g}\right)}{\partial \mathbf{h}_{k}}=2\left(g_{k j}\left(\mathbf{h}_{k} ; \boldsymbol{\theta}_{k}^{g}\right)-y_{k}\right) \frac{\partial g_{k j}\left(\mathbf{h}_{k} ; \boldsymbol{\theta}_{k}^{g}\right)}{\partial \mathbf{h}_{k}} \\
& \frac{\partial \log P\left(y_{k j} \mid \mathbf{h}_{k} ; \boldsymbol{\theta}_{k}^{g}\right)}{\partial \boldsymbol{\theta}_{k}^{g}}=2\left(g_{k j}\left(\mathbf{h}_{k} ; \boldsymbol{\theta}_{k}^{g}\right)-y_{k}\right) \frac{\partial g_{k j}\left(\mathbf{h}_{k} ; \boldsymbol{\theta}_{k}^{g}\right)}{\partial \boldsymbol{\theta}_{k}^{g}}
\end{aligned}
$$

- Transition layer

$$
\frac{\partial \mathbf{h}_{k}}{\partial \mathbf{h}_{k-1}}=\frac{\partial f_{k}\left(\mathbf{h}_{k-1}, x_{k} ; \boldsymbol{\theta}_{k}^{h}\right)}{\partial \mathbf{h}_{k-1}}, \frac{\partial \mathbf{h}_{k}}{\partial \boldsymbol{\theta}_{k}^{h}}=\frac{\partial f_{k}\left(\mathbf{h}_{k-1}, x_{k} ; \boldsymbol{\theta}_{k}^{h}\right)}{\partial \boldsymbol{\theta}_{k}^{h}}, \frac{\partial \mathbf{h}_{k}}{\partial \mathbf{x}_{k}}=\frac{\partial f_{k}\left(\mathbf{h}_{k-1}, \mathbf{x}_{k} ; \boldsymbol{\theta}_{k}^{h}\right)}{\partial \mathbf{x}_{k}}
$$

Depending on the different function forms of $f_{k}(\cdot)$ and $g_{k}(\cdot)$, the gradient can be computed.

\section{E Multi-task Elastic Net}

In this section, we will briefly review the multi-task elastic net methods in the literature. Here, $Y \in \mathbb{R}^{n \times k}$ is the multivariate output, where $n$ is the number of the samples and $k$ is the number of tasks. $X \in \mathbb{R}^{n \times p}$ is the input variables and $p$ is the number of feature variables. $W \in \mathbb{R}^{p \times k}$ is the regression coefficient matrix. 
Finally, to impose the similarity between multiple tasks, the following regularization is used as follows.

$$
\frac{1}{2 n}\|Y-X W\|^{2}+\alpha \beta\|W\|_{2,1}+\frac{1}{2} \alpha(1-\beta)\|W\|^{2} .
$$

Here, $\|W\|_{2,1}=\sum_{i} \sqrt{\sum_{j} W_{i j}^{2}}$ is the sum of norm of each row of $W$, where $\|W\|_{2,1}$ encourages the same sparsity patterns among all tasks. For example, it is easy to show that when the number of tasks equals to one, the problem MEN reduces to the $L_{1}$-norm regularized optimization problem (or Lasso). When there are multiple tasks, the weights corresponding to the $i$-th feature are grouped together via the $L_{2}$-norm of $W$. Furthermore, $\|W\|^{2}$ is used to further regularize the model to avoid the multicollinearity of the task input matrix $X . \alpha$ and $\beta$ are two tuning parameters control the level of regularization.

\section{References}

Abellan-Nebot, J. V., Liu, J., Subiron, F. R., and Shi, J. (2012). State space modeling of variation propagation in multistation machining processes considering machining-induced variations. Journal of Manufacturing Science and Engineering, 134(2):021002.

Apley, D. and Shi, J. (1998). Diagnosis of multiple fixture faults in panel assembly. Journal of Manufacturing Science and Engineering, 120(4):793-801.

Apley, D. W. (2016). Visualizing the effects of predictor variables in black box supervised learning models. arXiv preprint arXiv:1612.08468.

Bakır, B., Batmaz, İ., Güntürkün, F., İpekçi, İ., Köksal, G., and Özdemirel, N. (2006). Defect cause modeling with decision tree and regression analysis. World Acad Sci Eng Technoly, 24:1-4.

Bottou, L. (2010). Large-scale machine learning with stochastic gradient descent. In Proceedings of COMPSTAT'2010, pages 177-186. Springer.

Caruana, R. (1997). Multitask learning. Machine learning, 28(1):41-75.

Ceglarek, D. and Shi, J. (1996). Fixture failure diagnosis for autobody assembly using pattern recognition. Journal of Engineering for Industry, 118(1):55-66.

Chang, D. S. and Jiang, S.-T. (2002). Assessing quality performance based on the on-line sensor measurements using neural networks. Computers $\&$ industrial engineering, 42(2-4):417-424. 
Correa, M., Bielza, C., Ramirez, M. d. J., and Alique, J. R. (2008). A bayesian network model for surface roughness prediction in the machining process. International Journal of Systems Science, 39(12):11811192.

Ding, Y., Jin, J., Ceglarek, D., and Shi, J. (2005). Process-oriented tolerancing for multi-station assembly systems. iiE Transactions, 37(6):493-508.

Friedman, N., Geiger, D., and Goldszmidt, M. (1997). Bayesian network classifiers. Machine learning, 29(2-3):131-163.

Graves, A., Mohamed, A.-r., and Hinton, G. (2013a). Speech recognition with deep recurrent neural networks. In Acoustics, speech and signal processing (icassp), ieee international conference on, pages 6645-6649. IEEE.

Graves, A., Mohamed, A.-r., and Hinton, G. (2013b). Speech recognition with deep recurrent neural networks. In 2013 IEEE international conference on acoustics, speech and signal processing, pages 6645-6649. IEEE.

Graves, A. and Schmidhuber, J. (2009). Offline handwriting recognition with multidimensional recurrent neural networks. In Advances in neural information processing systems, pages 545-552.

Hao, L., Bian, L., Gebraeel, N., and Shi, J. (2016). Residual life prediction of multistage manufacturing processes with interaction between tool wear and product quality degradation. IEEE Transactions on Automation Science and Engineering.

Huang, Q., Zhou, S., and Shi, J. (2002). Diagnosis of multi-operational machining processes through variation propagation analysis. Robotics and Computer-Integrated Manufacturing, 18(3-4):233-239.

Jemwa, G. T. and Aldrich, C. (2005). Improving process operations using support vector machines and decision trees. AIChE journal, 51(2):526-543.

Jensen, F. V. (1996). An introduction to Bayesian networks, volume 210. UCL press London.

Jin, J. and Shi, J. (1999). State space modeling of sheet metal assembly for dimensional control. Journal of Manufacturing Science and Engineering, 121(4):756-762.

Jin, M. and Tsung, F. (2009). A chart allocation strategy for multistage processes. IIE Transactions, $41(9): 790-803$.

Jin, R., Li, J., and Shi, J. (2007). Quality prediction and control in rolling processes using logistic regression. Transactions of NAMRI/SME, 35:113-120. 
Jin, R. and Shi, J. (2012). Reconfigured piecewise linear regression tree for multistage manufacturing process control. IIE Transactions, 44(4):249-261.

Joe Qin, S. (2003). Statistical process monitoring: basics and beyond. Journal of Chemometrics: A Journal of the Chemometrics Society, 17(8-9):480-502.

Kourti, T., Lee, J., and Macgregor, J. F. (1996). Experiences with industrial applications of projection methods for multivariate statistical process control. Computers \& Chemical Engineering, 20:S745-S750.

Kuhn, M. and Johnson, K. (2013). Applied predictive modeling, volume 26. Springer.

Lawless, J., Mackay, R., and Robinson, J. (1999). Analysis of variation transmission in manufacturing processes-part i. Journal of Quality Technology, 31(2):131.

LeCun, Y., Boser, B. E., Denker, J. S., Henderson, D., Howard, R. E., Hubbard, W. E., and Jackel, L. D. (1990). Handwritten digit recognition with a back-propagation network. In Advances in neural information processing systems, pages 396-404.

Li, Y. and Tsung, F. (2009). False discovery rate-adjusted charting schemes for multistage process monitoring and fault identification. Technometrics, 51(2):186-205.

Liu, J., Shi, J., and Hu, S. J. (2009). Quality-assured setup planning based on the stream-of-variation model for multi-stage machining processes. IIE transactions, 41(4):323-334.

Liu, K. and Shi, J. (2013). Objective-oriented optimal sensor allocation strategy for process monitoring and diagnosis by multivariate analysis in a bayesian network. IIE Transactions, 45(6):630-643.

Liu, K., Zhang, X., and Shi, J. (2014). Adaptive sensor allocation strategy for process monitoring and diagnosis in a bayesian network. IEEE Transactions on Automation Science and Engineering, 11(2):452462.

Lowry, C. A. and Montgomery, D. C. (1995). A review of multivariate control charts. IIE transactions, 27(6):800-810.

MacGregor, J. F. and Kourti, T. (1995). Statistical process control of multivariate processes. Control Engineering Practice, 3(3):403-414.

Mateos, G. and Giannakis, G. B. (2011). Robust nonparametric regression via sparsity control with application to load curve data cleansing. IEEE Transactions on Signal Processing, 60(4):1571-1584.

Molnar, C. (2020). Interpretable Machine Learning. Lulu. com. 
Obozinski, G., Taskar, B., and Jordan, M. (2006). Multi-task feature selection. Statistics Department, UC Berkeley, Tech. Rep, 2(2.2).

Paynabar, K., Jin, J., and Pacella, M. (2013). Monitoring and diagnosis of multichannel nonlinear profile variations using uncorrelated multilinear principal component analysis. IIE transactions, 45(11):12351247.

Reckhow, K. H. (1999). Water quality prediction and probability network models. Canadian Journal of Fisheries and Aquatic Sciences, 56(7):1150-1158.

Shewhart, W. A. (1931). Economic control of quality of manufactured product. ASQ Quality Press.

Shi, J. (2006). Stream of variation modeling and analysis for multistage manufacturing processes. CRC press.

Shiu, B., Ceglarek, D., and Shi, J. (1997). Flexible beam-based modeling of sheet metal assembly for dimensional control. Transactions-North American Manufacturing Research Institution of SME, pages $49-54$.

Skinner, K. R., Montgomery, D. C., Runger, G. C., Fowler, J. W., McCarville, D. R., Rhoads, T. R., and Stanley, J. D. (2002). Multivariate statistical methods for modeling and analysis of wafer probe test data. IEEE transactions on semiconductor manufacturing, 15(4):523-530.

Tam, C., Tong, T. K., Lau, T. C., and Chan, K. (2004). Diagnosis of prestressed concrete pile defects using probabilistic neural networks. Engineering Structures, 26(8):1155-1162.

Woodall, W. H. (2007). Current research on profile monitoring. Production, 17(3):420-425.

Yan, H., Paynabar, K., and Pacella, M. (2018). Structured point cloud data analysis via regularized tensor regression for process modeling and optimization. Technometrics, (just-accepted):1-36.

Yan, H., Paynabar, K., and Shi, J. (2015). Image-based process monitoring using low-rank tensor decomposition. IEEE Transactions on Automation Science and Engineering, 12(1):216-227.

Yan, H., Paynabar, K., and Shi, J. (2017). Anomaly detection in images with smooth background via smooth-sparse decomposition. Technometrics, 59(1):102-114.

Yu, J. and Rashid, M. M. (2013). A novel dynamic bayesian network-based networked process monitoring approach for fault detection, propagation identification, and root cause diagnosis. AIChE Journal, $59(7): 2348-2365$. 
Yuan, M. and Lin, Y. (2006). Model selection and estimation in regression with grouped variables. Journal of the Royal Statistical Society: Series B (Statistical Methodology), 68(1):49-67.

Zhang, C., Yan, H., Lee, S., and Shi, J. (2018). Dynamic multivariate functional data modeling via sparse subspace learning. arXiv preprint arXiv:1804.0379\%.

Zhou, Q., Xiong, Z., Zhang, J., and Xu, Y. (2006). Hierarchical neural network based product quality prediction of industrial ethylene pyrolysis process. In International Symposium on Neural Networks, pages 1132-1137. Springer.

Zhou, S., Chen, Y., and Shi, J. (2004). Statistical estimation and testing for variation root-cause identification of multistage manufacturing processes. IEEE Transactions on Automation Science and Engineering, $1(1): 73-83$.

Zhou, S., Huang, Q., and Shi, J. (2003). State space modeling of dimensional variation propagation in multistage machining process using differential motion vectors. IEEE Transactions on robotics and automation, 19(2):296-309. 